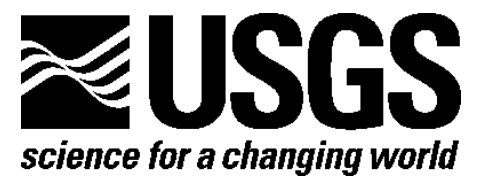

\title{
Multi-Elemental Analysis of Aqueous Geochemical Samples by Quadrupole Inductively Coupled Plasma-Mass Spectrometry (ICP-MS)
}

By Ruth E. Wolf and Monique Adams

Open-File Report 2015-1010 


\section{U.S. Department of the Interior \\ SALLY JEWELL, Secretary}

\section{U.S. Geological Survey \\ Suzette M. Kimball, Acting Director}

U.S. Geological Survey, Reston, Virginia: 2015

For more information on the USGS-the Federal source for science about the Earth, its natural and living resources, natural hazards, and the environment-visit http://www.usgs.gov/ or call 1-888-ASK-USGS (1-888-275-8747).

For an overview of USGS information products, including maps, imagery, and publications, visit http://www.usgs.gov/pubprod/.

Any use of trade, firm, or product names is for descriptive purposes only and does not imply endorsement by the U.S. Government.

Although this information product, for the most part, is in the public domain, it also may contain copyrighted materials as noted in the text. Permission to reproduce copyrighted items must be secured from the copyright owner.

Suggested citation:

Wolf, R.E., and Adams, Monique, 2015, Multi-elemental analysis of aqueous geochemical samples by quadrupole inductively coupled plasma-mass spectrometry (ICP-MS): U.S. Geological Survey Open-File Report 2015-1010, p. 34, http://dx.doi.org/10.3133/ofr20151010.

ISSN 2331-1258 (online) 


\section{Contents}

Principle

Interferences and Other Sources of Analytical Bias.................................................................................. 1

Scope

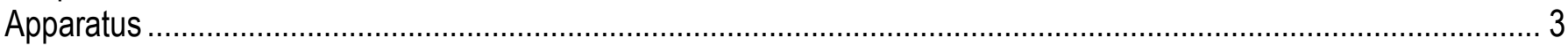

Reagents

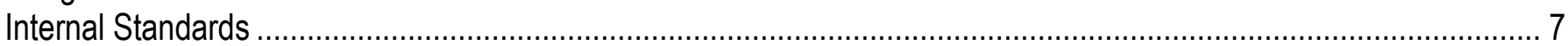

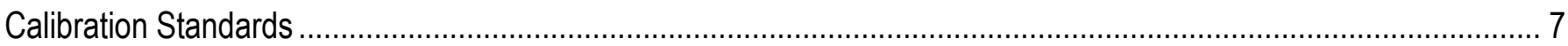

Safety Precautions

Sample Preparation

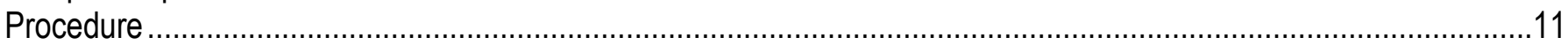

Method Performance —Blank Analysis and Reporting Limits...........................................................................13

Method Performance -Analyses of Quality Control Samples..............................................................................16

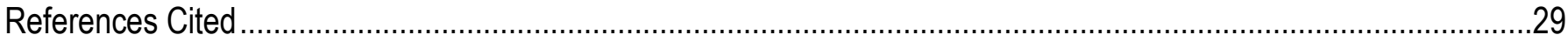

Appendix 1. Determination of Empirical Oxide Corrections ..................................................................................

\section{Tables}

1. Elements determined in aqueous samples by Inductively Coupled Plasma-Mass Spectrometry and determination parameters ................................................................................................................... 4

2. Attenuation levels achieved using Extended Dynamic Range mode ……..................................................... 7

3. Sources, elements, and concentrations of commercially available stock solutions ......................................... 8

4. Elements and concentrations of Water Matrix Stock solution ..................................................................... 9

5. Preparation and final concentration of calibration standards for Inductively Coupled Plasma-Mass

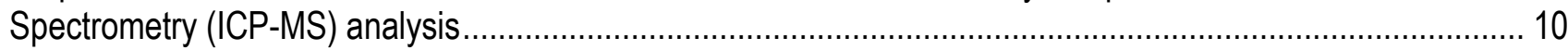

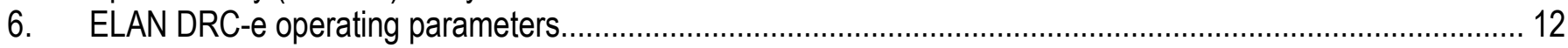

7. Preparation and final concentration of independent calibration verification standards for Inductively

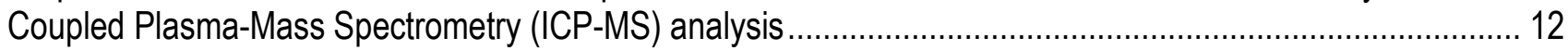

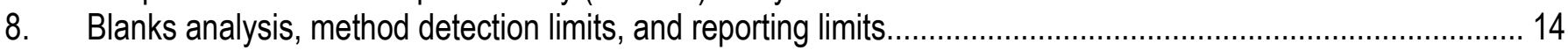

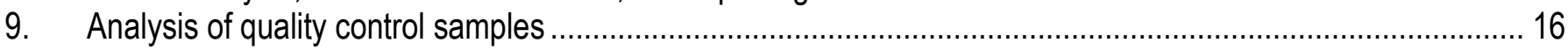

1-1. Elements and timing parameters for oxide interference correction determination............................................ 31

1-2. Correction equations calculation worksheet with formulas used for calculations shown .................................... 34 


\section{Conversion Factors}

Inch/Pound to SI

\begin{tabular}{|c|c|c|}
\hline Multiply & By & To obtain \\
\hline \multicolumn{3}{|c|}{ Length } \\
\hline inch (in.) & 2.54 & centimeter $(\mathrm{cm})$ \\
\hline inch (in.) & 25.4 & millimeter $(\mathrm{mm})$ \\
\hline \multicolumn{3}{|c|}{ Volume } \\
\hline ounce, fluid (fl. oz) & 0.02957 & liter $(\mathrm{L})$ \\
\hline $\operatorname{pint}(\mathrm{pt})$ & 0.4732 & liter (L) \\
\hline quart (qt) & 0.9464 & liter (L) \\
\hline gallon (gal) & 3.785 & liter (L) \\
\hline gallon (gal) & 0.003785 & cubic meter $\left(\mathrm{m}^{3}\right)$ \\
\hline cubic inch $\left(\mathrm{in}^{3}\right)$ & 16.39 & cubic centimeter $\left(\mathrm{cm}^{3}\right)$ \\
\hline cubic inch $\left(\mathrm{in}^{3}\right)$ & 0.01639 & liter $(\mathrm{L})$ \\
\hline \multicolumn{3}{|c|}{ Flow rate } \\
\hline foot per second (ft/s) & 0.3048 & meter per second $(\mathrm{m} / \mathrm{s})$ \\
\hline foot per minute $(\mathrm{ft} / \mathrm{min})$ & 0.3048 & meter per minute $(\mathrm{m} / \mathrm{min})$ \\
\hline \multicolumn{3}{|c|}{ Mass } \\
\hline ounce, avoirdupois (oz) & 28.35 & $\operatorname{gram}(\mathrm{g})$ \\
\hline pound, avoirdupois (lb) & 0.4536 & kilogram (kg) \\
\hline \multicolumn{3}{|c|}{ Pressure } \\
\hline atmosphere, standard (atm) & 101.3 & kilopascal (kPa) \\
\hline bar & 100 & kilopascal (kPa) \\
\hline \multicolumn{3}{|c|}{ Density } \\
\hline pound per cubic foot $\left(\mathrm{lb} / \mathrm{ft}^{3}\right)$ & 0.01602 & $\begin{array}{l}\text { gram per cubic centimeter } \\
\left(\mathrm{g} / \mathrm{cm}^{3}\right)\end{array}$ \\
\hline \multicolumn{3}{|c|}{ Energy } \\
\hline kilowatthour (kWh) & $3,600,000$ & joule $(\mathrm{J})$ \\
\hline
\end{tabular}

Specific conductance is given in microsiemens per centimeter at 25 degrees Celsius $\left(\mu \mathrm{S} / \mathrm{cm}\right.$ at $\left.25^{\circ} \mathrm{C}\right)$.

Concentrations of chemical constituents in water are given either in milligrams per liter $(\mathrm{mg} / \mathrm{L})$ or micrograms per liter $(\mu \mathrm{g} / \mathrm{L})$. 


\title{
Multi-Elemental Analysis of Aqueous Geochemical Samples by Quadrupole Inductively Coupled Plasma-Mass Spectrometry (ICP-MS)
}

\author{
By Ruth E. Wolf and Monique Adams
}

\section{Principle}

Typically, quadrupole inductively coupled plasma-mass spectrometry (ICP-MS) is used to determine as many as 57 major, minor, and trace elements in aqueous geochemical samples, including natural surface water and groundwater, acid mine drainage water, and extracts or leachates from geological samples. The sample solution is aspirated into the inductively coupled plasma (ICP) which is an electrodeless discharge of ionized argon gas at a temperature of approximately 6,000 degrees Celsius. The elements in the sample solution are subsequently volatilized, atomized, and ionized by the ICP. The ions generated are then focused and introduced into a quadrupole mass filter which only allows one mass to reach the detector at a given moment in time. As the settings of the mass analyzer change, subsequent masses are allowed to impact the detector. Although the typical quadrupole ICP-MS system is a sequential scanning instrument (determining each mass separately), the scan speed of modern instruments is on the order of several thousand masses per second. Consequently, typical total sample analysis times of 2-3 minutes are readily achievable for up to 57 elements.

For the protocol described, the ICP-MS is calibrated using a blank and a minimum of four standards prepared from commercially available multi-element standard solutions in conjunction with two standards for phosphorus and sulfur. At the discretion of the analyst, additional elements may be determined after suitable method modifications and performance data are established. Calibration curves are verified using a minimum of one standard prepared from a second commercial source and two reference water samples or certified reference materials obtained from a commercial source in a ready to analyze state. Samples to be analyzed for "dissolved" constituents must be filtered and acid-preserved in 1-2 percent nitric acid $\left(\mathrm{HNO}_{3}\right)$ at sample collection or as soon as possible after collection prior to analyses (no digestion is required). Samples to be analyzed for "total" elements require a digestion before analysis such as U.S. Environmental Protection Agency (EPA) Method 3015A-Microwave Assisted Acid Digestion for Aqueous Samples and Extracts (U.S. Environmental Protection Agency, 2007a). This method may also be used to analyze digested biological samples including insects, plants, and biological tissue.

\section{Interferences and Other Sources of Analytical Bias}

ICP-MS interferences come from spectroscopic and nonspectroscopic (or matrix) sources (Jarvis, 1992). Spectroscopic interferences include direct isobaric overlaps, where an isotope of one element exists at the same nominal mass as an isotope of another element, for example chromium and iron both have naturally occurring isotopes at mass $54\left({ }^{54} \mathrm{Cr}\right.$ and $\left.{ }^{54} \mathrm{Fe}\right)$. Polyatomic isobaric interferences may occur because of the formation of ions from plasma and sample matrix species; for example, ${ }^{40} \mathrm{Ar}$ from the plasma and ${ }^{35} \mathrm{Cl}$ from samples containing chloride or hydrochloric acid form ${ }^{40} \mathrm{Ar}^{35} \mathrm{Cl}^{+}$at mass 75 , interfering with the determination of arsenic at ${ }^{75} \mathrm{As}^{+}$. Double-charged 
interferences can occur when an element forms a double-charged species in the plasma and can interfere with elements at lower masses; for example, in high barium matrices, ${ }^{138} \mathrm{Ba}^{2+}$ formation in the plasma results in a signal at a mass-to-charge ratio of 69 , interfering with the determination of gallium at ${ }^{69} \mathrm{Ga}$. Refractory oxides can result from incomplete atomization of the sample matrix or recombination of the species in the plasma. Plasma tuning parameters such as radio frequency (RF) power and cooling, auxiliary, and sample argon flows are generally established during the instrument tuning process to minimize oxide formation; however, for some applications, such as the determination of rare earth elements (REEs), oxide formation is a serious source of interference and analytical bias. Commonly, the isotopes measured for each element to be determined are selected to minimize isobaric overlap from other elements and molecular species that may be present in the sample matrix or formed in the argon plasma. For some isotopes, isobaric overlap corrections are computed based on relative isotopic abundances of the elements involved, and oxide or double-charged ion intensities. For the REEs, empirical oxide correction equations are used that must be re-evaluated on a routine basis when instrument tuning parameters are changed. Instrumentation with Dynamic Reaction Cell (DRC) technology (Perkin Elmer, Inc., Shelton, Conn.) also has the capability to use different reaction gases in the DRC cell between the ICP ion source and the analyzer quadrupole to break apart and remove molecular interferences or to shift the analyte ion away from a nonremovable interference by selective gas phase reaction chemistry (Tanner and others, 2002).

Nonspectroscopic interferences include effects that cause suppression or enhancement of the analytical signal or physical effects caused by high total dissolved solids. Nonspectroscopic effects are commonly referred to as matrix effects, because they can vary with each sample matrix type. Matrix effects are often unpredictable and not well understood. Known matrix effects include viscosity differences between different types and (or) concentrations of acids that result in changes in sample uptake, nebulization, and vaporization rates (Olesik and Bates, 1995). Specific examples include signal enhancements observed in matrices containing organic solvents, signal suppression of low mass elements in solutions containing significant levels of high mass elements, signal enhancements of certain elements in high carbon-containing solutions, and suppression of some elements in the presence of easily ionizable elements such as Na, K, and Cs (Allain and others, 1991; Jarvis, 1992; Kralj and Veber, 2003). High levels of total dissolved solids can lead to partial clogging of the sample interface cones between the atmospheric ICP discharge and the high vacuum of the mass analyzer and result in significant levels of signal drift over time. Although matrix interferences can be minimized by careful matching of sample and calibration matrices, the use of internal standards is generally required to compensate for matrix effects and long-term instrumental drift (Lichte and others, 1987; Jarvis, 1992; Horlick and Montaser, 1998). When internal standards are used, the ratio of the analyte intensity to the internal standard intensity is calculated and used in all subsequent calibration calculations.

Most aqueous samples are not digested prior to analysis, but usually they will have been filtered. Samples that have been filtered and acid preserved (FA) generally do not require additional filtering unless particulate matter has precipitated during storage and transport. Raw, acidified (RA) samples that have not been filtered at collection may contain particulate material because some elements will precipitate over time. At the analyst's discretion, these samples may be filtered (using $0.45 \mu \mathrm{m}$ disposable syringe filters) or decanted prior to analysis to prevent particulate matter from clogging the sample introduction system (for example, the sample probe or nebulizer). Samples with conductivities greater than $1,500 \mu \mathrm{S} / \mathrm{cm}$ or total dissolved solids (TDS) levels of greater than 0.1 percent are generally diluted by the analyst in order to prevent clogging of the sample introduction system and minimize matrix interferences. The analyst should notify the sample submitter if this or any other procedures were necessary to analyze the samples. 


\section{Scope}

ICP-MS has been applied to the determination of 57 elements in various aqueous matrices, including natural waters, acid mine drainage waters, and extract and leachate samples. Analytes for which EPA has demonstrated the acceptability of ICP-MS determinations for waters and waste extracts (U.S. Environmental Protection Agency, 2007b) are included in this work. In addition to the 23 EPA target analyte list (TAL) elements, 34 elements are included in this protocol per the provisions of Method 6020A, including the REEs. The elements analyzed, masses utilized, and interference correction equations utilized are shown in table 1.

Some major elements are determined using extended dynamic range (EDR) mode in order to limit the need to dilute samples. In EDR mode, the quadrupole inside the DRC is utilized in direct current (dc) voltage-only mode without a reaction cell gas to attenuate the ion beam passed through to the analyzer quadrupole (Tanner and Baranov, 1999; Abou-Shakar, F., 2005), resulting in higher linear ranges. In order to implement EDR mode, the elements expected to be at high concentrations in the samples are analyzed with the DRC parameter $\mathrm{RPa}$ at two or three nonzero values in addition to the normal mode value of $\mathrm{RPa}=0$ while the $\mathrm{RF}$-controlling parameter, $\mathrm{RPq}$, is held at the normal mode value of $R P q=0.25$. The values of $R P q$ and $R P a$ control the mass bandpass in the DRC. $R P q$ is the low mass cutoff and controls the RF applied to the quadrupole rods in the $\mathrm{DRC}$. RPa is analogous to a high mass cutoff and controls the dc voltage applied to the quadrupole rods in the DRC (Tanner and others, 2002). The elements in EDR mode can be determined at various RPa attenuation values within the same analytical run, eliminating the need to dilute most samples. Table 2 shows some examples of the levels of signal attenuation that can be obtained using the EDR mode.

\section{Apparatus}

- Quadrupole Inductively Coupled Plasma-Mass Spectrometer equipped with Dynamic Reaction Cell (PerkinElmer ELAN DRC-e, ELAN DRCII, NexION 300D, or NexION 350D are the models currently used, PerkinElmer Life and Analytical Sciences, Shelton, Conn.)

- Random access autosampler compatible with ICP-MS instrumentation listed above

- Peltier-cooled quartz spray chamber (Model PC-3, Elemental Scientific, Inc., Omaha, Nebr.) or quartz cyclonic spray chamber (PerkinElmer Life and Analytical Sciences, Shelton, Conn.)

- Glass or quartz concentric nebulizer, 0.5-1.0 milliliter per minute ( $\mathrm{mL} / \mathrm{min})$ uptake rate, A- or C-type (Meinhard Inc., Golden, Colo.)

- 13 x 100 millimeter $(\mathrm{mm})$ disposable, polypropylene test tubes with caps (VWR International, Radnor, Pa., vials part number 60818-860 and caps part number 60828-738)

- Adjustable mechanical or electronic pipettes, typical volume ranges needed are from 5 to 120 microliters $(\mu \mathrm{L})$, from 50 to $1,000 \mu \mathrm{L}$, and from 100 to $5,000 \mu \mathrm{L}$

- 15 and 50 milliliter (mL) BD Falcon polypropylene tubes (BD Biosciences, San Jose, Calif.)

- Peristaltic pump tubing, polyvinyl chloride (PVC) type (Meinhard Inc., Golden, Colo.):

- Internal standard: Orange-orange (0.89 $\mathrm{mm}$ inner diameter [i.d.])

- Sample: Orange-orange (0.89 mm i.d.)

○ Drain: Purple-purple (2.06 mm i.d.)

- Autosampler peristaltic pump tubing, Santoprene type: Red-red (1.14 mm i.d.), 3-stop

- Plastic mixing tee (1/16-in. i.d.) (Cole-Parmer, EW-30506-15) 
Table 1. Elements determined in aqueous samples by Inductively Coupled Plasma-Mass Spectrometry and determination parameters.

[cps, counts per second; MCA, multichannel analyzer; ms, milliseconds; EDR, extended dynamic range; IEC, interelement correction]

\begin{tabular}{|c|c|c|c|c|c|c|c|c|c|}
\hline $\begin{array}{l}\text { Internal } \\
\text { standard }\end{array}$ & Analyte & Mass & Scan mode & $\begin{array}{c}\text { MCA } \\
\text { channels }\end{array}$ & Dwell time & $\begin{array}{l}\text { Integration } \\
\text { time }\end{array}$ & Mode & Equations & $\begin{array}{l}\text { Equation } \\
\text { type }\end{array}$ \\
\hline & $\mathrm{Li}$ & 6.015 & Peak Hopping & 1 & $50.0 \mathrm{~ms}$ & $750 \mathrm{~ms}$ & Normal & & \\
\hline $6 \mathrm{Li}$ & $\mathrm{Li}$ & 7.016 & Peak Hopping & 1 & $50.0 \mathrm{~ms}$ & $750 \mathrm{~ms}$ & Normal & & \\
\hline $6 \mathrm{Li}$ & $\mathrm{Be}$ & 9.012 & Peak Hopping & 1 & $50.0 \mathrm{~ms}$ & $750 \mathrm{~ms}$ & Normal & & \\
\hline $103 \mathrm{Rh}$ & $\mathrm{Na}$ & 22.99 & Peak Hopping & 1 & $50.0 \mathrm{~ms}$ & $750 \mathrm{~ms}$ & Normal & & \\
\hline $103 \mathrm{Rh}$ & $\mathrm{Na}-1$ & 22.99 & Peak Hopping & 1 & $50.0 \mathrm{~ms}$ & $750 \mathrm{~ms}$ & $\begin{array}{c}\text { EDR - } \\
\mathrm{Rpa}=0.014\end{array}$ & & \\
\hline $103 \mathrm{Rh}$ & $\mathrm{Na}-2$ & 22.99 & Peak Hopping & 1 & $50.0 \mathrm{~ms}$ & $750 \mathrm{~ms}$ & $\begin{array}{c}\text { EDR - } \\
\mathrm{Rpa}=0.016\end{array}$ & & \\
\hline $103 \mathrm{Rh}$ & $\mathrm{Mg}$ & 24.986 & Peak Hopping & 1 & $50.0 \mathrm{~ms}$ & $750 \mathrm{~ms}$ & Normal & & \\
\hline $103 \mathrm{Rh}$ & $\mathrm{Al}$ & 26.982 & Peak Hopping & 1 & $20.0 \mathrm{~ms}$ & $300 \mathrm{~ms}$ & Normal & & \\
\hline $103 \mathrm{Rh}$ & Al-1 & 26.982 & Peak Hopping & 1 & $50.0 \mathrm{~ms}$ & $750 \mathrm{~ms}$ & $\begin{array}{c}\text { EDR - } \\
\mathrm{Rpa}=0.012\end{array}$ & & \\
\hline $103 \mathrm{Rh}$ & $\mathrm{Si}$ & 28.977 & Peak Hopping & 1 & $20.0 \mathrm{~ms}$ & $300 \mathrm{~ms}$ & Normal & & \\
\hline $103 \mathrm{Rh}$ & $\mathrm{P}$ & 30.994 & Peak Hopping & 1 & $20.0 \mathrm{~ms}$ & $300 \mathrm{~ms}$ & Normal & & \\
\hline $103 \mathrm{Rh}$ & $\mathrm{S}$ & 33.968 & Peak Hopping & 1 & $50.0 \mathrm{~ms}$ & $750 \mathrm{~ms}$ & Normal & & \\
\hline $103 \mathrm{Rh}$ & K & 38.964 & Peak Hopping & 1 & $20.0 \mathrm{~ms}$ & $300 \mathrm{~ms}$ & Normal & & \\
\hline $103 \mathrm{Rh}$ & $\mathrm{Ca}$ & 42.959 & Peak Hopping & 1 & $20.0 \mathrm{~ms}$ & $300 \mathrm{~ms}$ & Normal & & \\
\hline $103 \mathrm{Rh}$ & $\mathrm{Ca}$ & 43.956 & Peak Hopping & 1 & $20.0 \mathrm{~ms}$ & $300 \mathrm{~ms}$ & Normal & & \\
\hline $103 \mathrm{Rh}$ & $\mathrm{Sc}$ & 44.956 & Peak Hopping & 1 & $20.0 \mathrm{~ms}$ & $300 \mathrm{~ms}$ & Normal & & \\
\hline $103 \mathrm{Rh}$ & $\mathrm{Ti}$ & 48.948 & Peak Hopping & 1 & $20.0 \mathrm{~ms}$ & $300 \mathrm{~ms}$ & Normal & & \\
\hline $103 \mathrm{Rh}$ & V & 50.944 & Peak Hopping & 1 & $20.0 \mathrm{~ms}$ & $300 \mathrm{~ms}$ & Normal & $-3.127 *(\mathrm{ClO} 53-(0.113 * \mathrm{Cr} 52))$ & Isobaric \\
\hline $103 \mathrm{Rh}$ & $\mathrm{Cr}$ & 51.941 & Peak Hopping & 1 & $20.0 \mathrm{~ms}$ & $300 \mathrm{~ms}$ & Normal & & \\
\hline $103 \mathrm{Rh}$ & $\mathrm{Cr}$ & 52.941 & Peak Hopping & 1 & $20.0 \mathrm{~ms}$ & $300 \mathrm{~ms}$ & Normal & & \\
\hline $103 \mathrm{Rh}$ & $\mathrm{Fe}$ & 53.94 & Peak Hopping & 1 & $20.0 \mathrm{~ms}$ & $300 \mathrm{~ms}$ & Normal & $-0.028226 * \operatorname{Cr} 52$ & Isobaric \\
\hline $103 \mathrm{Rh}$ & $\mathrm{Mn}$ & 54.938 & Peak Hopping & 1 & $20.0 \mathrm{~ms}$ & $300 \mathrm{~ms}$ & Normal & & \\
\hline $103 \mathrm{Rh}$ & $\mathrm{Fe}$ & 56.935 & Peak Hopping & 1 & $20.0 \mathrm{~ms}$ & $300 \mathrm{~ms}$ & Normal & $-0.049304 * \mathrm{Ca} 43$ & Empirical-IEC \\
\hline
\end{tabular}


Table 1. Elements determined in aqueous samples by Inductively Coupled Plasma-Mass Spectrometry and determination parameters. - Continued [cps, counts per second; MCA, multichannel analyzer; ms, milliseconds; EDR, extended dynamic range; IEC, interelement correction]

\begin{tabular}{|c|c|c|c|c|c|c|c|c|c|}
\hline $\begin{array}{l}\text { Internal } \\
\text { standard }\end{array}$ & Analyte & Mass & Scan mode & $\begin{array}{c}\text { MCA } \\
\text { channels }\end{array}$ & Dwell time & $\begin{array}{l}\text { Integration } \\
\text { time }\end{array}$ & Mode & Equations & $\begin{array}{l}\text { Equation } \\
\text { type }\end{array}$ \\
\hline $103 \mathrm{Rh}$ & $\mathrm{Ni}$ & 57.935 & Peak Hopping & 1 & $20.0 \mathrm{~ms}$ & $300 \mathrm{~ms}$ & Normal & $-0.0428 * \mathrm{Fe} 54-0.0037 * \mathrm{Ca} 43$ & Empirical-IEC \\
\hline $103 \mathrm{Rh}$ & Co & 58.933 & Peak Hopping & 1 & $20.0 \mathrm{~ms}$ & $300 \mathrm{~ms}$ & Normal & $-0.001231 * \mathrm{Ca} 43$ & Empirical-IEC \\
\hline $103 \mathrm{Rh}$ & $\mathrm{Ni}$ & 59.933 & Peak Hopping & 1 & $50.0 \mathrm{~ms}$ & $750 \mathrm{~ms}$ & Normal & $-0.0025 * \mathrm{Ca} 43$ & Empirical-IEC \\
\hline $103 \mathrm{Rh}$ & $\mathrm{Cu}$ & 64.928 & Peak Hopping & 1 & $20.0 \mathrm{~ms}$ & $300 \mathrm{~ms}$ & Normal & & \\
\hline $103 \mathrm{Rh}$ & $\mathrm{Zn}$ & 65.926 & Peak Hopping & 1 & $20.0 \mathrm{~ms}$ & $300 \mathrm{~ms}$ & Normal & $-0.0026^{*} \mathrm{Ti} 49$ & Isobaric \\
\hline $103 \mathrm{Rh}$ & $\mathrm{Ga}$ & 70.925 & Peak Hopping & 1 & $20.0 \mathrm{~ms}$ & $300 \mathrm{~ms}$ & Normal & & \\
\hline $103 \mathrm{Rh}$ & $\mathrm{Ge}$ & 73.922 & Peak Hopping & 1 & $20.0 \mathrm{~ms}$ & $300 \mathrm{~ms}$ & Normal & $-0.116645 *$ Se 77 & Isobaric \\
\hline $103 \mathrm{Rh}$ & As & 74.922 & Peak Hopping & 1 & $100.0 \mathrm{~ms}$ & $1500 \mathrm{~ms}$ & Normal & & \\
\hline $103 \mathrm{Rh}$ & As-1 & 74.922 & Peak Hopping & 1 & $100.0 \mathrm{~ms}$ & $1500 \mathrm{~ms}$ & Normal & $-3.127 *(\mathrm{ArCl} 77-0.825 * \mathrm{Se} 82)$ & Isobaric \\
\hline $103 \mathrm{Rh}$ & $\mathrm{Se}$ & 76.92 & Peak Hopping & 1 & $100.0 \mathrm{~ms}$ & $1500 \mathrm{~ms}$ & Normal & & \\
\hline $103 \mathrm{Rh}$ & $\mathrm{Se}$ & 81.917 & Peak Hopping & 1 & $100.0 \mathrm{~ms}$ & $1500 \mathrm{~ms}$ & Normal & $-1.007833 * \mathrm{Kr} 83$ & Isobaric \\
\hline $103 \mathrm{Rh}$ & $\mathrm{Rb}$ & 84.912 & Peak Hopping & 1 & $20.0 \mathrm{~ms}$ & $300 \mathrm{~ms}$ & Normal & & \\
\hline $103 \mathrm{Rh}$ & $\mathrm{Sr}$ & 87.906 & Peak Hopping & 1 & $20.0 \mathrm{~ms}$ & $300 \mathrm{~ms}$ & Normal & & \\
\hline $103 \mathrm{Rh}$ & $\mathrm{Y}$ & 88.905 & Peak Hopping & 1 & $20.0 \mathrm{~ms}$ & $300 \mathrm{~ms}$ & Normal & & \\
\hline $103 \mathrm{Rh}$ & $\mathrm{Zr}$ & 89.904 & Peak Hopping & 1 & $20.0 \mathrm{~ms}$ & $300 \mathrm{~ms}$ & Normal & & \\
\hline $103 \mathrm{Rh}$ & $\mathrm{Nb}$ & 92.906 & Peak Hopping & 1 & $20.0 \mathrm{~ms}$ & $300 \mathrm{~ms}$ & Normal & & \\
\hline \multirow[t]{2}{*}{$103 \mathrm{Rh}$} & Mo & 97.906 & Peak Hopping & 1 & $20.0 \mathrm{~ms}$ & $300 \mathrm{~ms}$ & Normal & $-0.110588 * \mathrm{Ru} 101$ & Isobaric \\
\hline & $\mathrm{Rh}$ & 102.905 & Peak Hopping & 1 & $20.0 \mathrm{~ms}$ & $300 \mathrm{~ms}$ & Normal & & \\
\hline $103 \mathrm{Rh}$ & $\mathrm{Ag}$ & 108.905 & Peak Hopping & 1 & $20.0 \mathrm{~ms}$ & $300 \mathrm{~ms}$ & Normal & $-0.006819 * \mathrm{Nb} 93$ & Isobaric \\
\hline $103 \mathrm{Rh}$ & $\mathrm{Cd}$ & 113.904 & Peak Hopping & 1 & $20.0 \mathrm{~ms}$ & $300 \mathrm{~ms}$ & Normal & $-0.027250 * \operatorname{Sn} 118$ & Isobaric \\
\hline $103 \mathrm{Rh}$ & $\mathrm{Sb}$ & 120.904 & Peak Hopping & 1 & $20.0 \mathrm{~ms}$ & $300 \mathrm{~ms}$ & Normal & & \\
\hline $103 \mathrm{Rh}$ & Cs & 132.905 & Peak Hopping & 1 & $20.0 \mathrm{~ms}$ & $300 \mathrm{~ms}$ & Normal & & \\
\hline $103 \mathrm{Rh}$ & $\mathrm{Ba}$ & 134.906 & Peak Hopping & 1 & $20.0 \mathrm{~ms}$ & $300 \mathrm{~ms}$ & Normal & & \\
\hline $193 \mathrm{Ir}$ & $\mathrm{La}$ & 138.906 & Peak Hopping & 1 & $20.0 \mathrm{~ms}$ & $300 \mathrm{~ms}$ & Normal & & \\
\hline
\end{tabular}


Table 1. Elements determined in aqueous samples by Inductively Coupled Plasma-Mass Spectrometry and determination parameters. - Continued [cps, counts per second; MCA, multichannel analyzer; ms, milliseconds; EDR, extended dynamic range; IEC, interelement correction]

\begin{tabular}{|c|c|c|c|c|c|c|c|c|c|}
\hline $\begin{array}{l}\text { Internal } \\
\text { standard }\end{array}$ & Analyte & Mass & Scan mode & $\begin{array}{c}\text { MCA } \\
\text { channels }\end{array}$ & Dwell time & $\begin{array}{l}\text { Integration } \\
\text { time }\end{array}$ & Mode & Equations & $\begin{array}{l}\text { Equation } \\
\text { type }\end{array}$ \\
\hline $193 \mathrm{Ir}$ & $\mathrm{Ce}$ & 139.905 & Peak Hopping & 1 & $20.0 \mathrm{~ms}$ & $300 \mathrm{~ms}$ & Normal & & \\
\hline $193 \mathrm{Ir}$ & $\operatorname{Pr}$ & 140.907 & Peak Hopping & 1 & $10.0 \mathrm{~ms}$ & $150 \mathrm{~ms}$ & Normal & & \\
\hline $193 \mathrm{Ir}$ & $\mathrm{Nd}$ & 145.913 & Peak Hopping & 1 & $10.0 \mathrm{~ms}$ & $150 \mathrm{~ms}$ & Normal & $-0.00034 * \mathrm{Ba} 135$ & Empirical-IEC \\
\hline $193 \mathrm{Ir}$ & $\mathrm{Sm}$ & 148.917 & Peak Hopping & 1 & $10.0 \mathrm{~ms}$ & $150 \mathrm{~ms}$ & Normal & & \\
\hline $193 \mathrm{Ir}$ & $\mathrm{Eu}$ & 150.92 & Peak Hopping & 1 & $10.0 \mathrm{~ms}$ & $150 \mathrm{~ms}$ & Normal & $-0.001238 * \mathrm{Ba} 135$ & Empirical-IEC \\
\hline $193 \mathrm{Ir}$ & $\mathrm{Tb}$ & 158.925 & Peak Hopping & 1 & $10.0 \mathrm{~ms}$ & $150 \mathrm{~ms}$ & Normal & $-0.011207 * \mathrm{Nd} 146$ & Empirical-IEC \\
\hline $193 \mathrm{Ir}$ & $\mathrm{Gd}$ & 159.927 & Peak Hopping & 1 & $10.0 \mathrm{~ms}$ & $150 \mathrm{~ms}$ & Normal & $\begin{array}{c}-0.079197 * \mathrm{Dy} 164-0.0197 * \mathrm{Nd} 146- \\
0.000596 * \mathrm{Sm} 149\end{array}$ & Empirical-IEC \\
\hline $193 \mathrm{Ir}$ & Dy & 163.929 & Peak Hopping & 1 & $10.0 \mathrm{~ms}$ & $150 \mathrm{~ms}$ & Normal & $\begin{array}{c}-0.046589 * \operatorname{Er} 166-0.00542 * \mathrm{Nd} 146- \\
0.00359 * \mathrm{Sm} 149\end{array}$ & Empirical-IEC \\
\hline $193 \mathrm{Ir}$ & Ho & 164.93 & Peak Hopping & 1 & $10.0 \mathrm{~ms}$ & $150 \mathrm{~ms}$ & Normal & $-0.004782 * \operatorname{Sm} 149$ & Empirical-IEC \\
\hline $193 \mathrm{Ir}$ & $\mathrm{Er}$ & 165.93 & Peak Hopping & 1 & $10.0 \mathrm{~ms}$ & $150 \mathrm{~ms}$ & Normal & $\begin{array}{c}-0.005547 * \mathrm{Nd} 146-0.002507 * \mathrm{Sm} \\
149\end{array}$ & Empirical-IEC \\
\hline $193 \mathrm{Ir}$ & $\mathrm{Tm}$ & 168.934 & Peak Hopping & 1 & $10.0 \mathrm{~ms}$ & $150 \mathrm{~ms}$ & Normal & $-0.000804 * \operatorname{Eu} 151$ & Empirical-IEC \\
\hline $193 \mathrm{Ir}$ & $\mathrm{Yb}$ & 171.937 & Peak Hopping & 1 & $10.0 \mathrm{~ms}$ & $150 \mathrm{~ms}$ & Normal & $-0.008746 * \mathrm{Gd} 160$ & Empirical-IEC \\
\hline $193 \mathrm{Ir}$ & $\mathrm{Lu}$ & 174.941 & Peak Hopping & 1 & $10.0 \mathrm{~ms}$ & $150 \mathrm{~ms}$ & Normal & & \\
\hline $193 \mathrm{Ir}$ & $\mathrm{Ta}$ & 180.948 & Peak Hopping & 1 & $20.0 \mathrm{~ms}$ & $300 \mathrm{~ms}$ & Normal & $-0.005965 *$ Ho 165 & Empirical-IEC \\
\hline \multirow[t]{2}{*}{$193 \mathrm{Ir}$} & $\mathrm{W}$ & 183.951 & Peak Hopping & 1 & $20.0 \mathrm{~ms}$ & $300 \mathrm{~ms}$ & Normal & $-0.004947 * \operatorname{Er} 166$ & Empirical-IEC \\
\hline & Ir & 192.963 & Peak Hopping & 1 & $20.0 \mathrm{~ms}$ & $300 \mathrm{~ms}$ & Normal & & \\
\hline $193 \mathrm{Ir}$ & $\mathrm{Tl}$ & 204.975 & Peak Hopping & 1 & $50.0 \mathrm{~ms}$ & $750 \mathrm{~ms}$ & Normal & & \\
\hline $193 \mathrm{Ir}$ & $\mathrm{Pb}$ & 207.977 & Peak Hopping & 1 & $20.0 \mathrm{~ms}$ & $300 \mathrm{~ms}$ & Normal & $+1 * \mathrm{~Pb} 206+1 * \mathrm{~Pb} 207$ & Isobaric \\
\hline $193 \mathrm{Ir}$ & $\mathrm{Bi}$ & 208.98 & Peak Hopping & 1 & $20.0 \mathrm{~ms}$ & $300 \mathrm{~ms}$ & Normal & & \\
\hline $193 \mathrm{Ir}$ & Th & 232.038 & Peak Hopping & 1 & $20.0 \mathrm{~ms}$ & $300 \mathrm{~ms}$ & Normal & & \\
\hline $193 \mathrm{Ir}$ & $\mathrm{U}$ & 238.05 & Peak Hopping & 1 & $20.0 \mathrm{~ms}$ & $300 \mathrm{~ms}$ & Normal & & \\
\hline
\end{tabular}


Table 2. Attenuation levels achieved using Extended Dynamic Range mode.

[cps, counts per second; mg/L, milligrams per liter; $\mu \mathrm{g} / \mathrm{L}$, micrograms per liter; \%, percent]

\begin{tabular}{ccc}
\hline Rpa & Na cps (5 $\mathbf{~ g / L ~ s t a n d a r d ) ~}$ & Attenuation factor \\
\hline 0.000 & 35153179 & 0 \\
0.014 & 4127026 & $88.3 \%$ \\
0.016 & 128618 & $99.6 \%$ \\
\hline Rpa & Al cps $\mathbf{5 0} \boldsymbol{~} \mathbf{g} / \mathbf{L}$ standard) & Attenuation factor \\
\hline 0.000 & 330886 & 0 \\
0.012 & 178419 & $46.1 \%$ \\
\hline
\end{tabular}

\section{Reagents}

- Deionized (DI) water, ASTM Type I, 18 megaohm (MS) (Milli-Q, EMD Millipore, Billerica, Mass.)

- Nitric acid $\left(\mathrm{HNO}_{3}\right)$, concentrated (70 percent), sub-boiling distilled (Reagent grade $\mathrm{HNO}_{3}, \mathrm{VWR}$ International, Radnor, Pa., purified using Milestone DuoPur, Milestone, Shelton, Conn.) or ULTREX grade or equivalent

- Two percent $\mathrm{HNO}_{3}$ (vol/vol) - Dilute $40 \mathrm{~mL}$ concentrated $\mathrm{HNO}_{3}$ to $2,000 \mathrm{~mL}$ with DI water

\section{Internal Standards}

Prepare $2 \mathrm{~L}$ of a solution containing $500 \mu \mathrm{g} / \mathrm{L}^{6} \mathrm{Li}, 20 \mu \mathrm{g} / \mathrm{L} \mathrm{Rh}$, and $20 \mu \mathrm{g} / \mathrm{L}$ Ir by serial dilution of commercial 1,000 mg/L aqueous standards (High Purity Standards, Charleston, S.C.), using 2 percent $\mathrm{HNO}_{3}$. This solution is mixed in a 1:1 ratio with the sample to be analyzed using two channels of the instrument peristaltic pump equipped with a plastic mixing tee and two lines of the orange-orange $(0.89$ $\mathrm{mm}$ i.d.) tubing. The concentrations of the internal standard solution may be adjusted, if necessary, to give target signal intensities between 200,000 and 600,000 counts per second (cps) for each internal standard element.

\section{Calibration Standards}

All calibration standards, continuous calibration verification (CCV) standards, and independent calibration verification (ICV) standards are prepared by serial dilution of commercially available multielement ICP-MS stock solutions and custom blended matrix stock solutions. The sources, elements, and concentrations of the commercially available stock solutions are given in table 3 . The elements and concentrations of the Water Matrix Stock solution are given in table 4. The water matrix stock can either be prepared from single element ICP-grade $10,000 \mathrm{mg} / \mathrm{L}$ or $1,000 \mathrm{mg} / \mathrm{L}$ commercially available standards or custom ordered prepared at the concentrations listed in table 4. Two separate sources of the Water Matrix Stock solution are required, one for preparation of the calibration standards and the second for the preparation of the independent calibration verification solution(s). Currently used sources of these custom-prepared standards include High Purity Standards (Charleston, S.C.) and Inorganic Ventures, Inc., (Christiansburg, Va.). The water matrix stock solution is added to the trace element stock solutions to provide an increasing concentration of common water matrix elements, reducing possible matrix effects from easily ionizable elements on trace element signal intensities (Horlick and Montaser, 1998; Wolf and others, 2001). Other 1,000 mg/L stock standards used for calibration standard preparation are available commercially (High Purity Standards, Charleston, S.C.). The preparation of 
Table 3. Sources, elements, and concentrations of commercially available stock solutions.

$[\mathrm{mg} / \mathrm{L}$, milligram per liter $]$

\begin{tabular}{|c|c|c|c|c|c|c|c|}
\hline $\begin{array}{l}\text { Name } \\
\text { Source } \\
\text { Part number } \\
\text { Concentration }\end{array}$ & $\begin{array}{c}\text { Calibration stock A } \\
\text { High Purity } \\
\text { ICP-MS-68A } \\
10 \mathrm{mg} / \mathrm{L} \\
\end{array}$ & $\begin{array}{c}\text { Calibration stock B } \\
\text { High Purity } \\
\text { ICP-MS-68B } \\
10 \mathrm{mg} / \mathrm{L} \\
\end{array}$ & $\begin{array}{c}\text { ICV stock } 1 \mathrm{~A} \text { Inorganic } \\
\text { Ventures } \\
\text { IV-ICPMS-71A } \\
10 \mathrm{mg} / \mathrm{L} \\
\end{array}$ & $\begin{array}{c}\text { ICV stock 1B } \\
\text { Inorganic Ventures } \\
\text { IV-ICPMS-71B } \\
10 \mathrm{mg} / \mathrm{L} \\
\end{array}$ & $\begin{array}{l}\text { ICV stock 2A } \\
\text { PerkinElmer } \\
\text { N9300233 } \\
10 \mathrm{mg} / \mathrm{L} \\
\end{array}$ & $\begin{array}{l}\text { ICV stock 2B } \\
\text { PerkinElmer } \\
\text { N9300232 } \\
10 \mathrm{mg} / \mathrm{L} \\
\end{array}$ & $\begin{array}{l}\text { ICV stock 2C } \\
\text { PerkinElmer } \\
\text { N9300235 } \\
10 \mathrm{mg} / \mathrm{L} \\
\end{array}$ \\
\hline \multirow[t]{48}{*}{ Elements } & $\mathrm{Al}$ & $\mathrm{Ag}$ & $\mathrm{Ag}$ & $\mathrm{Ge}$ & $\mathrm{Ag}$ & $\mathrm{Ce}$ & B \\
\hline & As & $\mathrm{Ge}$ & $\mathrm{Al}$ & $\mathrm{Hf}$ & $\mathrm{Al}$ & Dy & $\mathrm{Ge}$ \\
\hline & B & $\mathrm{Hf}$ & As & Mo & As & Er & Mo \\
\hline & $\mathrm{Ba}$ & Mo & B & $\mathrm{Nb}$ & $\mathrm{Ba}$ & $\mathrm{Eu}$ & $\mathrm{Nb}$ \\
\hline & $\mathrm{Be}$ & $\mathrm{Nb}$ & $\mathrm{Ba}$ & $\mathrm{Sb}$ & $\mathrm{Be}$ & $\mathrm{Gd}$ & $\mathrm{P}$ \\
\hline & $\mathrm{Bi}$ & $\mathrm{Sb}$ & $\mathrm{Be}$ & $\mathrm{Si}$ & $\mathrm{Bi}$ & Ho & $\mathrm{Re}$ \\
\hline & $\mathrm{Ca}$ & $\mathrm{Si}$ & $\mathrm{Ca}$ & $\mathrm{Sn}$ & $\mathrm{Ca}$ & $\mathrm{La}$ & $\mathrm{S}$ \\
\hline & $\mathrm{Cd}$ & $\mathrm{Sn}$ & $\mathrm{Cd}$ & $\mathrm{Ta}$ & $\mathrm{Cd}$ & $\mathrm{Lu}$ & $\mathrm{Si}$ \\
\hline & $\mathrm{Ce}$ & $\mathrm{Ta}$ & $\mathrm{Ce}$ & $\mathrm{Te}$ & Co & $\mathrm{Nd}$ & $\mathrm{Ta}$ \\
\hline & Co & $\mathrm{Te}$ & Co & $\mathrm{Ti}$ & $\mathrm{Cr}$ & $\operatorname{Pr}$ & $\mathrm{Ti}$ \\
\hline & $\mathrm{Cr}$ & $\mathrm{Ti}$ & $\mathrm{Cr}$ & W & Cs & $\mathrm{Sc}$ & W \\
\hline & Cs & W & Cs & $\mathrm{Zr}$ & $\mathrm{Cu}$ & $\mathrm{Sm}$ & $\mathrm{Zr}$ \\
\hline & $\mathrm{Cu}$ & $\mathrm{Zr}$ & $\mathrm{Cu}$ & & $\mathrm{Fe}$ & $\mathrm{Tb}$ & \\
\hline & Dy & & Dy & & $\mathrm{Ga}$ & Th & \\
\hline & $\mathrm{Er}$ & & $\mathrm{Er}$ & & In & $\mathrm{Tm}$ & \\
\hline & $\mathrm{Eu}$ & & $\mathrm{Eu}$ & & $\mathrm{K}$ & $\mathrm{Y}$ & \\
\hline & $\mathrm{Fe}$ & & $\mathrm{Fe}$ & & $\mathrm{Li}$ & $\mathrm{Yb}$ & \\
\hline & $\mathrm{Ga}$ & & $\mathrm{Ga}$ & & $\mathrm{Mg}$ & & \\
\hline & $\mathrm{Gd}$ & & $\mathrm{Gd}$ & & $\mathrm{Mn}$ & & \\
\hline & Ho & & Ho & & $\mathrm{Na}$ & & \\
\hline & In & & $\mathrm{K}$ & & $\mathrm{Ni}$ & & \\
\hline & $\mathrm{K}$ & & $\mathrm{La}$ & & $\mathrm{Pb}$ & & \\
\hline & $\mathrm{La}$ & & $\mathrm{Lu}$ & & $\mathrm{Rb}$ & & \\
\hline & $\mathrm{Li}$ & & $\mathrm{Mg}$ & & $\mathrm{Se}$ & & \\
\hline & $\mathrm{Lu}$ & & $\mathrm{Mn}$ & & $\mathrm{Sr}$ & & \\
\hline & $\mathrm{Mg}$ & & $\mathrm{Na}$ & & $\mathrm{Tl}$ & & \\
\hline & $\mathrm{Mn}$ & & $\mathrm{Nd}$ & & $\mathrm{U}$ & & \\
\hline & $\mathrm{Na}$ & & $\mathrm{Ni}$ & & $\mathrm{V}$ & & \\
\hline & $\mathrm{Nd}$ & & $\mathrm{P}$ & & $\mathrm{Zn}$ & & \\
\hline & $\mathrm{Ni}$ & & $\mathrm{Pb}$ & & & & \\
\hline & $\mathrm{P}$ & & $\operatorname{Pr}$ & & & & \\
\hline & $\mathrm{Pb}$ & & $\mathrm{Rb}$ & & & & \\
\hline & $\operatorname{Pr}$ & & $\mathrm{S}$ & & & & \\
\hline & $\mathrm{Rb}$ & & $\mathrm{Se}$ & & & & \\
\hline & $\mathrm{Re}$ & & $\mathrm{Sm}$ & & & & \\
\hline & $\mathrm{Sc}$ & & $\mathrm{Sr}$ & & & & \\
\hline & $\mathrm{Se}$ & & Th & & & & \\
\hline & $\mathrm{Sm}$ & & $\mathrm{Tl}$ & & & & \\
\hline & $\mathrm{Sr}$ & & $\mathrm{Tm}$ & & & & \\
\hline & $\mathrm{Tb}$ & & $\mathrm{U}$ & & & & \\
\hline & Th & & $\mathrm{V}$ & & & & \\
\hline & $\mathrm{Tl}$ & & $\mathrm{Yb}$ & & & & \\
\hline & $\mathrm{Tm}$ & & $\mathrm{Zn}$ & & & & \\
\hline & $\mathrm{U}$ & & & & & & \\
\hline & $\mathrm{V}$ & & & & & & \\
\hline & $\mathrm{Y}$ & & & & & & \\
\hline & $\mathrm{Yb}$ & & & & & & \\
\hline & $\mathrm{Zn}$ & & & & & & \\
\hline
\end{tabular}


the calibration standards and final concentrations are given in table 5. Note that because of the high sodium matrix in the commercially available phosphate and sulfate $1,000 \mathrm{mg} / \mathrm{L}$ stock standards, 25 $\mathrm{mg} / \mathrm{L}$ of $\mathrm{Ca}$ is added to the final prepared phosphate and sulfate calibration standards to eliminate signal enhancement effects caused by Easily Ionizable Element (EIE) effects. Standards are generally prepared to final volume by weight using the following procedure: (1) tare an empty $50 \mathrm{~mL}$ centrifuge tube on the balance; (2) pipette the designated amount of all stock solutions into the centrifuge tube; (3) add 2 percent $\mathrm{HNO}_{3}$ (vol/vol) up to the final desired weight using a wash bottle containing 2 percent $\mathrm{HNO}_{3}$ prepared with sub-boiling distilled $\mathrm{HNO}_{3}$ [Note: for 2 percent (vol/vol) $\mathrm{HNO}_{3}$, the density is $1.0 \mathrm{~g}$ per $1.0 \mathrm{~mL}$. If higher acid concentrations are used, density corrections may be necessary to prepare final volumes by weight. The density of the acid concentration being used can be measured by determining the weight of $1.00 \mathrm{~mL}$ of the acid pipetted into a tared vessel].

Table 4. Elements and concentrations of Water Matrix Stock solution.

$[\mathrm{mg} / \mathrm{L}$, milligram per liter]

\begin{tabular}{cc}
\hline Element & $\begin{array}{c}\text { Concentration } \\
\text { (mg/L) }\end{array}$ \\
\hline $\mathrm{Na}$ & 250 \\
$\mathrm{Ca}$ & 250 \\
$\mathrm{Mg}$ & 50 \\
$\mathrm{~K}$ & 50 \\
$\mathrm{Si}$ & 50 \\
\hline
\end{tabular}


Table 5. Preparation and final concentration of calibration standards for Inductively Coupled Plasma-Mass Spectrometry (ICP-MS) analysis.

[cal., calibration; $\mu \mathrm{g} / \mathrm{L}$, microgram per liter; $\mathrm{mg} / \mathrm{L}$, milligram per liter; \%, percent; $\mathrm{mL}$, milliliter; $\mu \mathrm{L}$, microliter; g, gram; $\mathrm{mg}$, milligram]

\begin{tabular}{|c|c|c|c|}
\hline Cal. Standard 1 & Stock solution & Volume $(\mu \mathrm{L})$ & Final concentration $(\mu \mathrm{g} / \mathrm{L})$ \\
\hline & Cal. Standard 2 & $5 \mathrm{~mL}$ & $1 \mu \mathrm{g} / \mathrm{L}$ (all elements) \\
\hline & $2 \% \mathrm{HNO}_{3}$ & to $50 \mathrm{~mL}$ (or $50 \mathrm{~g}$ ) & \\
\hline \multirow[t]{4}{*}{ Cal. Standard 2} & Stock solution & Volume $(\mu \mathrm{L})$ & Final concentration $(\mu \mathrm{g} / \mathrm{L})$ \\
\hline & ICP-MS-68A (High Purity) & $50 \mu \mathrm{L}$ & $10 \mu \mathrm{g} / \mathrm{L}$ (all elements) \\
\hline & ICP-MS-68B (High Purity) & $50 \mu \mathrm{L}$ & $10 \mu \mathrm{g} / \mathrm{L}$ (all elements) \\
\hline & $2 \% \mathrm{HNO}_{3}$ & to $50 \mathrm{~mL}$ (or $50 \mathrm{~g}$ ) & \\
\hline \multirow[t]{5}{*}{ Cal. Standard 3} & Stock solution & Volume $(\mu \mathrm{L})$ & Final concentration $(\mu \mathrm{g} / \mathrm{L})$ \\
\hline & ICP-MS-68A (High Purity) & $250 \mu \mathrm{L}$ & $50 \mu \mathrm{g} / \mathrm{L}$ (all elements) \\
\hline & ICP-MS-68B (High Purity) & $250 \mu \mathrm{L}$ & $50 \mu \mathrm{g} / \mathrm{L}$ (all elements) \\
\hline & Water Matrix Stock & $1000 \mu \mathrm{L}$ & $5 \mathrm{mg} / \mathrm{L} \mathrm{Na}, \mathrm{Ca} ; 1 \mathrm{mg} / \mathrm{L} \mathrm{Mg}, \mathrm{K}, \mathrm{Si}$ \\
\hline & $2 \% \mathrm{HNO}_{3}$ & to $50 \mathrm{~mL}$ (or $50 \mathrm{~g}$ ) & \\
\hline \multirow[t]{5}{*}{ Cal. Standard 4} & Stock solution & Volume $(\mu \mathrm{L})$ & Final concentration $(\mu \mathrm{g} / \mathrm{L})$ \\
\hline & ICP-MS-68A (High Purity) & $500 \mu \mathrm{L}$ & $100 \mu \mathrm{g} / \mathrm{L}$ (all elements) \\
\hline & ICP-MS-68B (High Purity) & $500 \mu \mathrm{L}$ & $100 \mu \mathrm{g} / \mathrm{L}$ (all elements) \\
\hline & Water Matrix Stock & $5 \mathrm{~mL}$ & $25 \mathrm{mg} / \mathrm{L} \mathrm{Na}, \mathrm{Ca} ; 5 \mathrm{mg} / \mathrm{L} \mathrm{Mg}, \mathrm{K}, \mathrm{Si}$ \\
\hline & $2 \% \mathrm{HNO}_{3}$ & to $50 \mathrm{~mL}$ (or $50 \mathrm{~g}$ ) & \\
\hline \multirow[t]{5}{*}{ Cal. Standard 5} & Stock solution & Volume $(\mu \mathrm{L})$ & Final concentration $(\mu \mathrm{g} / \mathrm{L})$ \\
\hline & ICP-MS-68A (High Purity) & $500 \mu \mathrm{L}$ & $250 \mu \mathrm{g} / \mathrm{L}$ (all elements) \\
\hline & ICP-MS-68B (High Purity) & $500 \mu \mathrm{L}$ & $250 \mu \mathrm{g} / \mathrm{L}$ (all elements) \\
\hline & Water Matrix Stock & $4 \mathrm{~mL}$ & $50 \mathrm{mg} / \mathrm{L} \mathrm{Na}, \mathrm{Ca} ; 10 \mathrm{mg} / \mathrm{L} \mathrm{Mg}, \mathrm{K}, \mathrm{Si}$ \\
\hline & $2 \% \mathrm{HNO}_{3}$ & to $20 \mathrm{~mL}$ (or $20 \mathrm{~g}$ ) & \\
\hline Cal. Standard 6 & Stock solution & Volume $(\mu \mathrm{L})$ & Final concentration $(\mu \mathrm{g} / \mathrm{L})$ \\
\hline \multirow[t]{4}{*}{$\left(\mathrm{SO}_{4}\right.$ and $\mathrm{PO}_{4}$ Low $)$} & $1,000 \mathrm{mg} / \mathrm{L} \mathrm{PO}_{4}$ & $2.5 \mathrm{~mL}$ & $125 \mathrm{mg} / \mathrm{L} \mathrm{PO}_{4} / 40.8 \mathrm{mg} / \mathrm{L} \mathrm{P}(1)$ \\
\hline & $1,000 \mathrm{mg} / \mathrm{L} \mathrm{SO}_{4}$ & $2.5 \mathrm{~mL}$ & $125 \mathrm{mg} / \mathrm{L} \mathrm{SO}_{4} / 41.8 \mathrm{mg} / \mathrm{L} \mathrm{S}(2)$ \\
\hline & $10,000 \mathrm{mg} / \mathrm{L} \mathrm{Ca}$ & $50 \mu \mathrm{L}$ & $25 \mathrm{mg} / \mathrm{L} \mathrm{Ca}$ \\
\hline & $2 \% \mathrm{HNO}_{3}$ & to $20 \mathrm{~mL}$ (or $20 \mathrm{~g}$ ) & \\
\hline Cal. Standard 7 & Stock solution & Volume $(\mu \mathrm{L})$ & Final concentration $(\mu \mathrm{g} / \mathrm{L})$ \\
\hline \multirow[t]{4}{*}{$\left(\mathrm{SO}_{4}\right.$ and $\left.\mathrm{PO}_{4} \mathrm{High}\right)$} & $1,000 \mathrm{mg} / \mathrm{L} \mathrm{PO}_{4}$ & $5 \mathrm{~mL}$ & $250 \mathrm{mg} / \mathrm{L} \mathrm{PO}_{4} / 81.6 \mathrm{mg} / \mathrm{L} \mathrm{P}(1)$ \\
\hline & $1,000 \mathrm{mg} / \mathrm{L} \mathrm{SO}_{4}$ & $5 \mathrm{~mL}$ & $250 \mathrm{mg} / \mathrm{L} \mathrm{SO}_{4} / 83.6 \mathrm{mg} / \mathrm{L} \mathrm{S}(2)$ \\
\hline & $10,000 \mathrm{mg} / \mathrm{L} \mathrm{Ca}$ & $50 \mu \mathrm{L}$ & $25 \mathrm{mg} / \mathrm{L} \mathrm{Ca}$ \\
\hline & $2 \% \mathrm{HNO}_{3}$ & to $20 \mathrm{~mL}$ (or $20 \mathrm{~g}$ ) & \\
\hline
\end{tabular}

Note 1: $1,000 \mathrm{mg} / \mathrm{L} \mathrm{PO}_{4}$ is equivalent to $326.4 \mathrm{mg} / \mathrm{L} \mathrm{P}$

Note 2: $1,000 \mathrm{mg} / \mathrm{L} \mathrm{SO}_{4}$ is equivalent to $334.4 \mathrm{mg} / \mathrm{L} \mathrm{S}$ 


\section{Safety Precautions}

All laboratory personnel are required to wear safety glasses, gloves, closed toe shoes, and lab coats when working in the laboratory. Refer to the laboratory chemical hygiene plan and material safety data sheets (MSDSs) for specific precautions, effects of overexposure, and first-aid treatment for reagents used in the preparation procedure and operation of the ICP-MS system.

\section{Sample Preparation}

Samples need to be preserved with $\mathrm{HNO}_{3}$ to a $\mathrm{pH} \leq 2$ at sample collection or as soon as possible after sample collection. Most sampling protocols require that the samples are filtered and acidified in the field (Wanty and others, 1999; Wilde and others, 2004; U.S. Geological Survey, 2006). At the analyst's discretion, unfiltered samples collected as raw acidified (RA) may be filtered or decanted prior to analysis if visible particulates or solids are present to prevent clogging of the sample introduction system (for example, sample probe or nebulizer). Samples with conductivities greater than 1,500 $\mu \mathrm{S} / \mathrm{cm}$ are generally diluted by the analyst in 2 percent $\mathrm{HNO}_{3}$ using a suitable dilution factor to bring the conductivity down to $<1,500 \mu \mathrm{S} / \mathrm{cm}$ in order to prevent clogging of the sample introduction system and minimize matrix interferences. The analyst should notify the sample submitter if this or any other procedures were necessary to analyze the samples. If the sample results are over the calibration curve, dilution and reanalysis of the affected samples for the over range elements is required or a high-level standard at a concentration above the highest measured sample level is analyzed with results within 5 percent of the true or prepared value.

\section{Procedure}

After a 30-minute warm-up time, the ICP-MS instrument is optimized to meet manufacturer or USGS-specific operating parameters, including Autolens and dual-detector calibration according to the instructions in the ELAN version 3.4 ICP-MS software. The instrument is then calibrated using the standard solutions listed in table 5, and the operating parameters shown in table 6 . The operating parameters may vary slightly depending on the specific model ICP-MS, nebulizer, and spray chamber being used for analysis.

The ICP-MS is calibrated using a blank and the seven standards listed in table 5 at the beginning of each analytical session using a linear through zero calibration equation. The calibration blank should be prepared from the same source and at the same acid concentration used in the calibration standards. Blank subtraction is performed to negate the effect of the reagents when the blank is higher than the detection limit. The accuracy of the method is checked using a minimum of three aqueous quality control samples such as certified reference materials (CRMs) from the National Institutes for Standards and Technology (NIST, http://www.nist.gov) and various other reference materials suppliers or Standard Reference Samples (SRSs) available from the USGS Standard Reference Sample Project (http://bqs.usgs.gov/srs/). 
Table 6. ELAN DRC-e operating parameters.

$[R F$, radio frequency; $\mathrm{W}$, watts; $\mathrm{L} / \mathrm{min}$, liters per minute; $\mathrm{mm}$, millimeter; rpm, rotations per minute; i.d., internal diameter; $\mathrm{s}$, seconds; ms, milliseconds]

\begin{tabular}{ll}
\hline \multicolumn{1}{c}{ Instrument parameter } & \multicolumn{1}{c}{ Setting or type } \\
\hline RF power & $1350 \mathrm{~W}$ \\
Cool gas (L/min) & 18 \\
Auxilary gas (L/min) & 1.5 \\
Nebulizer gas (L/min) & $0.78-0.82$ \\
Injector & $2 \mathrm{~mm}$ quartz \\
Spray chamber & Cyclonic, quartz, Peltier-cooled (PC3, ESI, Inc., Omaha, Neb.) \\
Pump Speed & $10 \mathrm{rpm}$ \\
Pump Tubing & Orange-orange (0.89 mm i.d.) \\
Uptake Rate & $1 \mathrm{~mL} /$ min (sample and internal standard lines together) \\
Integration time (ms) & $10-100$ milliseconds \\
Nebulizer & Meinhard Concentric, Type TR-50-C1 (Meinhard, Inc., Golden, Colo.) \\
Rinse time (s) & 90 seconds at 10 rpm \\
Specific method name & HP_Waters.mth \\
\hline
\end{tabular}

Before running unknown samples, the calibration is verified using one or more independent calibration verification (ICV) standards that are prepared from a stock originating from a different supplier than the stock(s) used to prepare the calibration standards. The ICV solution should be prepared at a level near the mid-point of each the calibration curve for each element. To verify calibrations for all 57 elements present in the calibration solutions, three separate ICV solutions are typically used-see table 7. The measured ICV concentrations must be within \pm 10 percent of the true or prepared value. If the ICV results are not within acceptable limits, then the source of the error should be identified and corrected and the instrument recalibrated and the ICV solution(s) reanalyzed. Common errors include improperly prepared calibration standards or stock solutions and inadequate mixing of the internal standard solution with the sample solutions. The results for all quality control sample (CRM or SRS) analyses should be within \pm 20 percent of the certified or proposed values, including stated errors. If the results are not within \pm 20 percent of the stated values, the samples are prepared again and reanalyzed by ICP-MS. If the results are still not within acceptable values, the instrument and method parameters are checked and the instrument is recalibrated using freshly prepared calibration standards and the quality control samples are reanalyzed. For every 10 unknown samples, a blank, continuous calibration verification (CCV), and a quality control sample are analyzed. The CCV solution(s) are prepared from the same stock standards used to prepare the calibration standards and may be one or more of the calibration standards reanalyzed to verify continuing calibration accuracy. The measured CCV concentrations should be within \pm 10 percent of the true or prepared value. If the CCV values are not within limits, the instrument and method parameters are checked and the instrument is recalibrated using freshly prepared calibration standards and any affected quality control and unknown samples are reanalyzed. If the quality control sample is not within \pm 20 percent of the accepted value, the instrument and method parameters are checked and the instrument is recalibrated using freshly prepared calibration standards, and any affected quality control and unknown samples are reanalyzed. 
Table 7. Preparation and final concentration of independent calibration verification standards for Inductively Coupled Plasma-Mass Spectrometry (ICP-MS) analysis.

$[\mu \mathrm{g} / \mathrm{L}$, microgram per liter; mg/L, milligram per liter; \%, percent; $\mathrm{mL}$, milliliter; $\mu \mathrm{L}$, microliter; g, gram; mg, milligram]

\begin{tabular}{|c|c|c|c|}
\hline I.V. ICV-75 & Stock solution & Volume $(\mu \mathrm{L})$ & Final concentration $(\mu \mathrm{g} / \mathrm{L})$ \\
\hline & ICV Stock 1A (IV-ICPMS-71A) & $150 \mu \mathrm{L}$ & $75 \mu \mathrm{g} / \mathrm{L}$ (all elements) \\
\hline & ICV Stock 1B (IV-ICPMS-71B) & $150 \mu \mathrm{L}$ & $75 \mu \mathrm{g} / \mathrm{L}$ (all elements) \\
\hline & Second Source Water Matrix Stock & $2 \mathrm{~mL}$ & $25 \mathrm{mg} / \mathrm{L} \mathrm{Na}, \mathrm{Ca} ; 5 \mathrm{mg} / \mathrm{L} \mathrm{Mg}, \mathrm{K}, \mathrm{Si}$ \\
\hline & $2 \% \mathrm{HNO}_{3}$ & to $20 \mathrm{~mL}$ (or $20 \mathrm{~g}$ ) & \\
\hline \multirow[t]{6}{*}{ PE ICV-75 } & Stock solution & Volume $(\mu \mathrm{L})$ & Final concentration $(\mu \mathrm{g} / \mathrm{L})$ \\
\hline & ICV Stock 2A (N9300233) & $150 \mu \mathrm{L}$ & $75 \mu \mathrm{g} / \mathrm{L}$ (all elements) \\
\hline & ICV Stock 2B (N9300232) & $150 \mu \mathrm{L}$ & $75 \mu \mathrm{g} / \mathrm{L}$ (all elements) \\
\hline & ICV Stock 2C (N9300235) & $150 \mu \mathrm{L}$ & $75 \mu \mathrm{g} / \mathrm{L}$ (all elements) \\
\hline & Second Source Water Matrix Stock & $2 \mathrm{~mL}$ & $25 \mathrm{mg} / \mathrm{L} \mathrm{Na}, \mathrm{Ca} ; 5 \mathrm{mg} / \mathrm{L} \mathrm{Mg}, \mathrm{K}, \mathrm{Si}$ \\
\hline & $2 \% \mathrm{HNO}_{3}$ & to $20 \mathrm{~mL}$ (or $20 \mathrm{~g}$ ) & \\
\hline P-S ICV-50 & Stock solution & Volume $(\mu \mathrm{L})$ & Final concentration $(\mu \mathrm{g} / \mathrm{L})$ \\
\hline \multirow[t]{4}{*}{$\left(\mathrm{SO}_{4}\right.$ and $\left.\mathrm{PO}_{4} \mathrm{ICV}\right)$} & Second Source $1000 \mathrm{mg} / \mathrm{L} \mathrm{PO}_{4}$ & $3.0 \mathrm{~mL}$ & $150 \mathrm{mg} / \mathrm{L} \mathrm{PO}_{4} / 49.0 \mathrm{mg} / \mathrm{L} \mathrm{P}$ \\
\hline & Second Source $1000 \mathrm{mg} / \mathrm{L} \mathrm{SO}_{4}$ & $3.0 \mathrm{~mL}$ & $150 \mathrm{mg} / \mathrm{L} \mathrm{SO}_{4} / 50.2 \mathrm{mg} / \mathrm{L} \mathrm{S}$ \\
\hline & $10,000 \mathrm{mg} / \mathrm{L} \mathrm{Ca}$ & $50 \mu \mathrm{L}$ & $25 \mathrm{mg} / \mathrm{L} \mathrm{Ca}$ \\
\hline & $2 \% \mathrm{HNO}_{3}$ & to $20 \mathrm{~mL}$ (or $20 \mathrm{~g}$ ) & \\
\hline
\end{tabular}

\section{Method Performance-Blank Analysis and Reporting Limits}

The instrument detection limits and reporting limits calculated from more than 40 blank analyses are shown in table 8 . The preferred masses for reporting are selected based on background equivalent concentration (BEC), achieved detection limits, analytical performance of quality control samples, and minimal interferences at the particular mass for a particular sample matrix. For some elements, more than one mass is analyzed in order to rule out possible interferences in unusual samples. Prior to reporting, the results for $\mathrm{Si}$ and $\mathrm{S}$ are converted to $\mathrm{SiO}_{2}$ and $\mathrm{SO}_{4}$ using 2.139 and 2.995 conversion factors, respectively. In table 8 , the method detection limit (MDL) is calculated as a value equaling three times the standard deviation (SD) of a large number $(n>40)$ of blank analyses during a 3-month period. The lower reporting limit in table 8 is established by rounding the calculated MDL to a suitable number of significant figures and raising reporting levels for ubiquitous elements based on professional judgment to prevent false positives. The instrument MDLs and reporting limits are reevaluated on an annual basis or as instrument operating parameters are changed. The upper reporting limit is generally extended to two times the highest calibration standard. For samples with results reading above the highest calibration standard, either a quality control sample at a higher level or a high-level calibration standard can be used to verify linearity above the highest calibration concentration. 
Table 8. Blanks analysis, method detection limits, and reporting limits.

$[\mu \mathrm{g} / \mathrm{L}$, microgram per liter; $\mathrm{mg} / \mathrm{L}$, milligram per liter; $\mathrm{SD}$, standard deviation; MDL, method detection limit]

\begin{tabular}{|c|c|c|c|c|c|c|c|c|}
\hline Element & Mass & $\begin{array}{c}\text { Calibration } \\
\text { units }\end{array}$ & $\begin{array}{c}\text { Number } \\
\text { of } \\
\text { samples }\end{array}$ & Mean & $\begin{array}{l}\text { Standard } \\
\text { deviation } \\
\text { (SD) }\end{array}$ & $\begin{array}{c}\text { MDL }=3 x \\
\text { SD }\end{array}$ & $\begin{array}{c}\text { Reporting } \\
\text { limit } \\
(\mu g / L)\end{array}$ & $\begin{array}{c}\text { Upper } \\
\text { reporting limit } \\
\text { (1) }\end{array}$ \\
\hline $\mathrm{Li}$ & 7 & $\mu \mathrm{g} / \mathrm{L}$ & 42 & -0.1243 & 0.8166 & 2.450 & 5 & 500 \\
\hline $\mathrm{Be}$ & 9 & $\mu \mathrm{g} / \mathrm{L}$ & 42 & -0.0008 & 0.0066 & 0.020 & 0.05 & 500 \\
\hline $\mathrm{Na}$ & 23 & $\mathrm{mg} / \mathrm{L}$ & 42 & 0.0026 & 0.0122 & 0.037 & 0.05 & 100 \\
\hline $\mathrm{Na}-1$ & 23 & $\mathrm{mg} / \mathrm{L}$ & 42 & 0.0025 & 0.0122 & 0.036 & 0.05 & 100 \\
\hline $\mathrm{Na}-2$ & 23 & $\mathrm{mg} / \mathrm{L}$ & 42 & 0.0027 & 0.0136 & 0.041 & 0.05 & 100 \\
\hline $\mathrm{Mg}$ & 25 & $\mathrm{mg} / \mathrm{L}$ & 42 & 0.0004 & 0.0030 & 0.009 & 0.01 & 20 \\
\hline $\mathrm{Al}$ & 27 & $\mu \mathrm{g} / \mathrm{L}$ & 42 & 0.3403 & 0.7442 & 2.233 & 3 & 500 \\
\hline Al-1 & 27 & $\mu \mathrm{g} / \mathrm{L}$ & 42 & 0.4815 & 0.7503 & 2.251 & 3 & 500 \\
\hline $\mathrm{Si}(2)$ & 29 & $\mathrm{mg} / \mathrm{L}$ & 42 & 0.0419 & 0.0670 & 0.201 & 0.2 & 40 \\
\hline $\mathrm{P}$ & 31 & $\mathrm{mg} / \mathrm{L}$ & 42 & 0.0022 & 0.0032 & 0.009 & 0.01 & 200 \\
\hline S (3) & 34 & $\mathrm{mg} / \mathrm{L}$ & 42 & 0.5845 & 1.6279 & 4.884 & 5 & 200 \\
\hline $\mathrm{K}$ & 39 & $\mathrm{mg} / \mathrm{L}$ & 42 & 0.0039 & 0.0065 & 0.019 & 0.03 & 20 \\
\hline $\mathrm{Ca}$ & 43 & $\mathrm{mg} / \mathrm{L}$ & 42 & 0.0002 & 0.0234 & 0.070 & 0.2 & 100 \\
\hline $\mathrm{Ca}$ & 44 & $\mathrm{mg} / \mathrm{L}$ & 42 & 0.0024 & 0.0105 & 0.032 & 0.2 & 100 \\
\hline Sc & 45 & $\mu \mathrm{g} / \mathrm{L}$ & 42 & 0.0358 & 0.1111 & 0.333 & 0.6 & 500 \\
\hline $\mathrm{Ti}$ & 49 & $\mu \mathrm{g} / \mathrm{L}$ & 42 & -0.0024 & 0.0473 & 0.142 & 0.5 & 500 \\
\hline V & 51 & $\mu \mathrm{g} / \mathrm{L}$ & 42 & -0.0012 & 0.0237 & 0.071 & 0.5 & 500 \\
\hline $\mathrm{Cr}$ & 52 & $\mu \mathrm{g} / \mathrm{L}$ & 42 & 0.0060 & 0.0913 & 0.274 & 1 & 500 \\
\hline $\mathrm{Cr}$ & 53 & $\mu \mathrm{g} / \mathrm{L}$ & 42 & 0.0185 & 0.2494 & 0.748 & 1 & 500 \\
\hline $\mathrm{Fe}$ & 54 & $\mu \mathrm{g} / \mathrm{L}$ & 42 & 3.1744 & 6.6543 & 19.963 & 60 & 500 \\
\hline $\mathrm{Mn}$ & 55 & $\mu \mathrm{g} / \mathrm{L}$ & 42 & -0.0014 & 0.0074 & 0.022 & 0.2 & 500 \\
\hline $\mathrm{Fe}$ & 57 & $\mu \mathrm{g} / \mathrm{L}$ & 42 & -4.7339 & 19.6910 & 59.073 & 60 & 500 \\
\hline $\mathrm{Ni}$ & 58 & $\mu \mathrm{g} / \mathrm{L}$ & 42 & -0.0374 & 0.0896 & 0.269 & 0.3 & 500 \\
\hline $\mathrm{Co}$ & 59 & $\mu \mathrm{g} / \mathrm{L}$ & 42 & 0.0001 & 0.0011 & 0.003 & 0.4 & 500 \\
\hline $\mathrm{Ni}$ & 60 & $\mu \mathrm{g} / \mathrm{L}$ & 42 & -0.0125 & 0.0739 & 0.222 & 0.4 & 500 \\
\hline $\mathrm{Cu}$ & 63 & $\mu \mathrm{g} / \mathrm{L}$ & 42 & -0.0030 & 0.0128 & 0.038 & 0.5 & 500 \\
\hline $\mathrm{Zn}$ & 66 & $\mu \mathrm{g} / \mathrm{L}$ & 42 & 0.0359 & 0.0619 & 0.186 & 1 & 500 \\
\hline $\mathrm{Ga}$ & 71 & $\mu \mathrm{g} / \mathrm{L}$ & 42 & -0.0002 & 0.0027 & 0.008 & 0.05 & 500 \\
\hline $\mathrm{Ge}$ & 74 & $\mu \mathrm{g} / \mathrm{L}$ & 42 & 0.0002 & 0.0038 & 0.011 & 0.05 & 500 \\
\hline As-1 & 75 & $\mu \mathrm{g} / \mathrm{L}$ & 42 & -0.0229 & 0.0654 & 0.196 & 1 & 500 \\
\hline As & 75 & $\mu \mathrm{g} / \mathrm{L}$ & 42 & -0.0042 & 0.0264 & 0.079 & 1 & 500 \\
\hline $\mathrm{Se}$ & 77 & $\mu \mathrm{g} / \mathrm{L}$ & 42 & 0.0800 & 0.2186 & 0.656 & 1 & 500 \\
\hline $\mathrm{Se}$ & 82 & $\mu \mathrm{g} / \mathrm{L}$ & 42 & -0.0059 & 0.1515 & 0.455 & 1 & 500 \\
\hline $\mathrm{Rb}$ & 85 & $\mu \mathrm{g} / \mathrm{L}$ & 42 & 0.0010 & 0.0018 & 0.005 & 0.01 & 500 \\
\hline $\mathrm{Sr}$ & 88 & $\mu \mathrm{g} / \mathrm{L}$ & 42 & 0.0096 & 0.0191 & 0.057 & 0.5 & 500 \\
\hline $\mathrm{Y}$ & 89 & $\mu \mathrm{g} / \mathrm{L}$ & 42 & 0.0002 & 0.0008 & 0.002 & 0.01 & 500 \\
\hline $\mathrm{Zr}$ & 90 & $\mu \mathrm{g} / \mathrm{L}$ & 42 & 0.0021 & 0.0031 & 0.009 & 0.2 & 500 \\
\hline $\mathrm{Nb}$ & 93 & $\mu \mathrm{g} / \mathrm{L}$ & 42 & 0.0100 & 0.0097 & 0.029 & 0.2 & 500 \\
\hline
\end{tabular}


Table 8. Blanks analysis, method detection limits, and reporting limits.-Continued

$[\mu \mathrm{g} / \mathrm{L}$, microgram per liter; $\mathrm{mg} / \mathrm{L}$, milligram per liter; SD, standard deviation; MDL, method detection limit]

\begin{tabular}{|c|c|c|c|c|c|c|c|c|}
\hline Element & Mass & $\begin{array}{l}\text { Calibration } \\
\text { units }\end{array}$ & $\begin{array}{l}\text { Number } \\
\text { of } \\
\text { samples }\end{array}$ & Mean & $\begin{array}{l}\text { Standard } \\
\text { deviation } \\
\text { (SD) }\end{array}$ & $\begin{array}{c}M D L=3 x \\
S D\end{array}$ & $\begin{array}{l}\text { Reporting } \\
\quad \text { limit } \\
(\mu \mathrm{g} / \mathrm{L})\end{array}$ & $\begin{array}{c}\text { Upper } \\
\text { reporting limit } \\
\text { (1) }\end{array}$ \\
\hline Mo & 98 & $\mu \mathrm{g} / \mathrm{L}$ & 42 & -0.0225 & 0.0901 & 0.270 & 2 & 500 \\
\hline $\mathrm{Ag}$ & 109 & $\mu \mathrm{g} / \mathrm{L}$ & 42 & -0.0501 & 0.0884 & 0.265 & 1 & 500 \\
\hline $\mathrm{Cd}$ & 114 & $\mu \mathrm{g} / \mathrm{L}$ & 42 & 0.0003 & 0.0011 & 0.003 & 0.02 & 500 \\
\hline $\mathrm{Sb}$ & 121 & $\mu \mathrm{g} / \mathrm{L}$ & 42 & 0.0188 & 0.0178 & 0.053 & 0.3 & 500 \\
\hline Cs & 133 & $\mu \mathrm{g} / \mathrm{L}$ & 42 & 0.0009 & 0.0010 & 0.003 & 0.02 & 500 \\
\hline $\mathrm{Ba}$ & 135 & $\mu \mathrm{g} / \mathrm{L}$ & 42 & 0.0036 & 0.0127 & 0.038 & 0.2 & 500 \\
\hline $\mathrm{La}$ & 139 & $\mu \mathrm{g} / \mathrm{L}$ & 42 & 0.0003 & 0.0007 & 0.002 & 0.01 & 500 \\
\hline $\mathrm{Ce}$ & 140 & $\mu \mathrm{g} / \mathrm{L}$ & 42 & 0.0002 & 0.0008 & 0.002 & 0.01 & 500 \\
\hline $\operatorname{Pr}$ & 141 & $\mu \mathrm{g} / \mathrm{L}$ & 42 & 0.0004 & 0.0005 & 0.002 & 0.01 & 500 \\
\hline $\mathrm{Nd}$ & 146 & $\mu \mathrm{g} / \mathrm{L}$ & 42 & 0.0008 & 0.0015 & 0.004 & 0.01 & 500 \\
\hline $\mathrm{Sm}$ & 149 & $\mu \mathrm{g} / \mathrm{L}$ & 42 & 0.0008 & 0.0017 & 0.005 & 0.01 & 500 \\
\hline $\mathrm{Eu}$ & 151 & $\mu \mathrm{g} / \mathrm{L}$ & 42 & 0.0003 & 0.0008 & 0.002 & 0.005 & 500 \\
\hline $\mathrm{Tb}$ & 159 & $\mu \mathrm{g} / \mathrm{L}$ & 42 & 0.0003 & 0.0006 & 0.002 & 0.005 & 500 \\
\hline $\mathrm{Gd}$ & 160 & $\mu \mathrm{g} / \mathrm{L}$ & 42 & 0.0006 & 0.0012 & 0.004 & 0.005 & 500 \\
\hline Dy & 164 & $\mu \mathrm{g} / \mathrm{L}$ & 42 & 0.0002 & 0.0014 & 0.004 & 0.005 & 500 \\
\hline Ho & 165 & $\mu \mathrm{g} / \mathrm{L}$ & 42 & 0.0004 & 0.0006 & 0.002 & 0.005 & 500 \\
\hline Er & 166 & $\mu \mathrm{g} / \mathrm{L}$ & 42 & -0.0002 & 0.0010 & 0.003 & 0.005 & 500 \\
\hline $\mathrm{Tm}$ & 168 & $\mu \mathrm{g} / \mathrm{L}$ & 42 & 0.0002 & 0.0005 & 0.002 & 0.005 & 500 \\
\hline $\mathrm{Yb}$ & 172 & $\mu \mathrm{g} / \mathrm{L}$ & 42 & -0.0001 & 0.0013 & 0.004 & 0.01 & 500 \\
\hline $\mathrm{Lu}$ & 175 & $\mu \mathrm{g} / \mathrm{L}$ & 42 & 0.0002 & 0.0004 & 0.001 & 0.1 & 500 \\
\hline $\mathrm{Ta}$ & 181 & $\mu \mathrm{g} / \mathrm{L}$ & 42 & 0.0150 & 0.0130 & 0.039 & 0.05 & 500 \\
\hline W & 184 & $\mu \mathrm{g} / \mathrm{L}$ & 42 & 0.0320 & 0.0278 & 0.084 & 0.5 & 500 \\
\hline $\mathrm{Tl}$ & 205 & $\mu \mathrm{g} / \mathrm{L}$ & 42 & 0.0064 & 0.0073 & 0.022 & 0.1 & 500 \\
\hline $\mathrm{Pb}$ & 208 & $\mu \mathrm{g} / \mathrm{L}$ & 42 & 0.0005 & 0.0034 & 0.010 & 0.05 & 500 \\
\hline $\mathrm{Bi}$ & 209 & $\mu \mathrm{g} / \mathrm{L}$ & 42 & 0.0033 & 0.0045 & 0.013 & 0.2 & 500 \\
\hline Th & 232 & $\mu \mathrm{g} / \mathrm{L}$ & 42 & 0.0019 & 0.0019 & 0.006 & 0.2 & 500 \\
\hline $\mathrm{U}$ & 238 & $\mu \mathrm{g} / \mathrm{L}$ & 42 & 0.0000 & 0.0009 & 0.003 & 0.1 & 500 \\
\hline
\end{tabular}

(1) Upper limit is based on 2 times the highest calibration standard. Samples higher than this should be verified by running a check standard higher than the highest reading sample(s).

(2) multiply by 2.139 to report as $\mathrm{SiO}_{2}$

(3) multiply by 2.995 factor to report as $\mathrm{SO}_{4}$ 


\section{Method Performance-Analyses of Quality Control Samples}

Results obtained for repeated analyses of six quality control samples analyzed by this method (T-131, T-201, T-207, T-175, M-158, and PPREE) are summarized in table 9. These samples, in addition to ICV samples discussed in the ICP-MS analyses section, are used to check the method performance during analyses of unknown samples that range from pristine natural waters to mine waste waters and various geologic sample leachates - samples that vary greatly in concentrations of trace and major elements. The six quality control samples shown in this report represent trace and major elements in natural waters collected at various locations in Colorado in which the trace and major constituents are modified (USGS Standard Reference Sample Project [http://bqs.usgs.gov/srs/]). The accepted or most probable value (MPV) for element concentrations in the USGS Standard Reference Sample (SRS) waters are determined via multilaboratory round-robin analysis. For many elements in the USGS SRSs, the levels are near the detection limits and are useful for evaluating accuracy at low concentrations. The PPREE sample is a standard reference water sample developed using mine water collected in the western San Juan Mountains, Colo. (Verplanck and others, 2001) that has elevated levels of Al, Ca, Fe, $\mathrm{Mg}, \mathrm{Mn}, \mathrm{Sr}$, and rare earth elements. Many of the interferences discussed in Appendix 1 affect accurate determination of these elements, and the obtained concentrations listed in table 9 are within \pm 20 percent of the accepted value for most elements in this quality control sample.

Table 9. Analysis of quality control samples.

[ $\mu \mathrm{g} / \mathrm{L}$, microgram per liter; mg/L, milligram per liter; NA, not applicable; $\mathrm{n}$, number; \%, percent; SD, standard deviation]

\begin{tabular}{|c|c|c|c|c|c|c|c|}
\hline $\begin{array}{l}\text { Quality Contol } \\
\text { Sample }\end{array}$ & & Description & $\mathrm{n}$ & $\begin{array}{c}\text { Average } \\
\text { determined } \\
\text { value }\end{array}$ & SD & $\begin{array}{l}\text { Accepted } \\
\text { value }\end{array}$ & $\%$ Recovery \\
\hline \multirow[t]{9}{*}{ Silver, Ag } & \multicolumn{2}{|c|}{$\mu \mathrm{g} / \mathrm{L}$} & & & & & \\
\hline & $\mathrm{T}-131$ & trace constituents & 29 & 1.149 & 0.34 & 1.26 & $91 \%$ \\
\hline & $\mathrm{T}-201$ & trace constituents & 34 & 17.75 & 4.11 & 17.30 & $103 \%$ \\
\hline & $\mathrm{T}-207$ & trace constituents & 18 & $<1$ & NA & 0.05 & NA \\
\hline & M-194 & major constituents & 27 & $<1$ & NA & NA & NA \\
\hline & M-198 & major constituents & 33 & $<1$ & NA & NA & NA \\
\hline & M-202 & major constituents & 23 & $<1$ & NA & NA & NA \\
\hline & PPREE-1 & trace constituents & 10 & $<1$ & NA & NA & NA \\
\hline & SCREE-1 & trace constituents & 10 & $<1$ & NA & NA & NA \\
\hline \multirow[t]{9}{*}{ Aluminum, $\mathrm{Al}$} & \multicolumn{2}{|c|}{$\mu \mathrm{g} / \mathrm{L}$} & & & & & \\
\hline & $\mathrm{T}-131$ & trace constituents & 29 & 128 & 7.00 & 132 & $97 \%$ \\
\hline & $\mathrm{T}-201$ & trace constituents & 34 & 77.7 & 5.40 & 77.8 & $100 \%$ \\
\hline & $\mathrm{T}-207$ & trace constituents & 14 & 320 & 12.0 & 324 & $99 \%$ \\
\hline & M-194 & major constituents & 27 & $<3$ & NA & NA & NA \\
\hline & M-198 & major constituents & 33 & $<3$ & NA & NA & NA \\
\hline & M-202 & major constituents & 23 & $<3$ & NA & NA & NA \\
\hline & PPREE-1 & trace constituents & 10 & 12180 & 600 & 11500 & $106 \%$ \\
\hline & SCREE-1 & trace constituents & 10 & 12600 & 650 & 11200 & $113 \%$ \\
\hline
\end{tabular}


Table 9. Analysis of quality control samples.-Continued

[ $\mu \mathrm{g} / \mathrm{L}$, microgram per liter; mg/L, milligram per liter; NA, not applicable; n, number; \%, percent; SD, standard deviation]

\begin{tabular}{|c|c|c|c|c|c|c|c|}
\hline $\begin{array}{l}\text { Quality Contol } \\
\text { Sample }\end{array}$ & & Description & $\mathrm{n}$ & $\begin{array}{c}\text { Average } \\
\text { determined } \\
\text { value }\end{array}$ & SD & $\begin{array}{l}\text { Accepted } \\
\text { value }\end{array}$ & $\%$ Recovery \\
\hline \multirow[t]{8}{*}{ Arsenic, As } & & & & & & & \\
\hline & $\mathrm{T}-131$ & trace constituents & 29 & 56.8 & 1.30 & 56.6 & $100 \%$ \\
\hline & $\mathrm{T}-201$ & trace constituents & 34 & 26.4 & 0.70 & 24.4 & $108 \%$ \\
\hline & $\mathrm{T}-207$ & trace constituents & 13 & 0.98 & 0.08 & 0.87 & $114 \%$ \\
\hline & M-198 & major constituents & 33 & $<0.3$ & NA & NA & NA \\
\hline & M-202 & major constituents & 23 & $<0.3$ & NA & NA & NA \\
\hline & PPREE-1 & trace constituents & 10 & 1.5 & 0.30 & 1.5 & $100 \%$ \\
\hline & SCREE-1 & trace constituents & 10 & $<0.3$ & NA & 0.2 & NA \\
\hline & $\mathrm{T}-207$ & trace constituents & 13 & 43.6 & 1.67 & 42.9 & $102 \%$ \\
\hline & M-194 & major constituents & 27 & 41.1 & 8.70 & NA & NA \\
\hline & M-198 & major constituents & 33 & 14.4 & 2.70 & NA & NA \\
\hline & M-202 & major constituents & 23 & 14.8 & 0.40 & NA & NA \\
\hline & PPREE-1 & trace constituents & 10 & 7.49 & 0.38 & 7.10 & $106 \%$ \\
\hline & SCREE-1 & trace constituents & 10 & 37.6 & 1.30 & 34.5 & $109 \%$ \\
\hline \multirow[t]{4}{*}{ Beryllium, Be } & & & & & & & \\
\hline & $\mathrm{T}-131$ & trace constituents & 29 & 12.2 & 0.90 & 12.2 & $100 \%$ \\
\hline & $\mathrm{T}-201$ & trace constituents & 34 & 8.29 & 0.74 & 8.35 & $99 \%$ \\
\hline & SCREE-1 & trace constituents & 10 & $<0.5$ & NA & 0.4 & NA \\
\hline \multirow[t]{9}{*}{ Bismuth, Bi } & & & & & & & \\
\hline & $\mathrm{T}-131$ & trace constituents & 29 & 14.4 & 1.13 & NA & NA \\
\hline & $\mathrm{T}-201$ & trace constituents & 34 & $<0.2$ & 0.01 & NA & NA \\
\hline & $\mathrm{T}-207$ & trace constituents & 18 & $<0.2$ & 0.00 & NA & NA \\
\hline & M-194 & major constituents & 27 & $<0.2$ & 0.02 & NA & NA \\
\hline & M-198 & major constituents & 32 & $<0.2$ & 0.01 & NA & NA \\
\hline & M-202 & major constituents & 22 & $<0.2$ & 0.01 & NA & NA \\
\hline & PPREE-1 & trace constituents & 10 & $<0.2$ & 0.00 & NA & NA \\
\hline & SCREE-1 & trace constituents & 10 & $<0.2$ & 0.00 & NA & NA \\
\hline
\end{tabular}


Table 9. Analysis of quality control samples.-Continued

[ $\mu \mathrm{g} / \mathrm{L}$, microgram per liter; mg/L, milligram per liter; NA, not applicable; $n$, number; \%, percent; SD, standard deviation]

\begin{tabular}{|c|c|c|c|c|c|c|c|}
\hline $\begin{array}{l}\text { Quality Contol } \\
\text { Sample }\end{array}$ & & Description & $\mathrm{n}$ & $\begin{array}{c}\text { Average } \\
\text { determined } \\
\text { value }\end{array}$ & SD & $\begin{array}{l}\text { Accepted } \\
\text { value }\end{array}$ & $\%$ Recovery \\
\hline \multirow[t]{9}{*}{ Calcium, $\mathrm{Ca}$} & & & & & & & \\
\hline & $\mathrm{T}-131$ & trace constituents & 29 & 31.6 & 1.50 & 30.6 & $103 \%$ \\
\hline & $\mathrm{T}-201$ & trace constituents & 34 & 53.7 & 2.70 & 53.1 & $101 \%$ \\
\hline & $\mathrm{T}-207$ & trace constituents & 18 & 23.5 & 1.10 & 23.4 & $100 \%$ \\
\hline & M-194 & major constituents & 27 & 23.3 & 5.50 & 24.4 & $95 \%$ \\
\hline & M-198 & major constituents & 32 & 10.2 & 0.61 & 10.4 & $98 \%$ \\
\hline & M-202 & major constituents & 22 & 5.5 & 0.20 & 5.6 & $98 \%$ \\
\hline & PPREE-1 & trace constituents & 10 & 481 & 56.3 & NA & NA \\
\hline & SCREE-1 & trace constituents & 10 & 95.6 & 9.50 & NA & NA \\
\hline \multirow[t]{9}{*}{ Cadmium, Cd } & & & & & & & \\
\hline & $\mathrm{T}-131$ & trace constituents & 29 & 6.6 & 0.34 & 6.1 & $108 \%$ \\
\hline & $\mathrm{T}-201$ & trace constituents & 34 & 16.7 & 0.57 & 15.7 & $106 \%$ \\
\hline & $\mathrm{T}-207$ & trace constituents & 18 & 0.63 & 0.03 & 0.59 & $107 \%$ \\
\hline & M-194 & major constituents & 27 & $<0.02$ & 0.01 & NA & NA \\
\hline & M-198 & major constituents & 32 & $<0.02$ & 0.00 & NA & NA \\
\hline & M-202 & major constituents & 22 & $<0.02$ & 0.00 & NA & NA \\
\hline & PPREE-1 & trace constituents & 10 & 0.5 & 0.02 & NA & NA \\
\hline & SCREE-1 & trace constituents & 10 & 12.5 & 0.39 & NA & NA \\
\hline \multirow[t]{9}{*}{ Cerium, $\mathrm{Ce}$} & & & & & & & \\
\hline & $\mathrm{T}-131$ & trace constituents & 29 & 0.01 & 0.01 & NA & NA \\
\hline & $\mathrm{T}-201$ & trace constituents & 34 & 2.8 & 0.28 & NA & NA \\
\hline & $\mathrm{T}-207$ & trace constituents & 18 & 3.8 & 0.14 & NA & NA \\
\hline & M-194 & major constituents & 27 & $<0.01$ & 0.01 & NA & NA \\
\hline & M-198 & major constituents & 32 & $<0.01$ & 0.01 & NA & NA \\
\hline & M-202 & major constituents & 22 & $<0.01$ & 0.01 & NA & NA \\
\hline & PPREE-1 & trace constituents & 10 & 181 & 7.20 & 161 & $112 \%$ \\
\hline & SCREE-1 & trace constituents & 10 & 26.3 & 1.20 & 24.6 & $107 \%$ \\
\hline \multirow[t]{9}{*}{ Cobalt, Co } & & & & & & & \\
\hline & $\mathrm{T}-131$ & trace constituents & 29 & 26.3 & 0.75 & 24.6 & $107 \%$ \\
\hline & $\mathrm{T}-201$ & trace constituents & 34 & 18.1 & 0.73 & 17.4 & $104 \%$ \\
\hline & $\mathrm{T}-207$ & trace constituents & 18 & 0.95 & 0.03 & 0.98 & $97 \%$ \\
\hline & M-194 & major constituents & 27 & $<0.02$ & 0.00 & NA & NA \\
\hline & M-198 & major constituents & 32 & $<0.02$ & 0.00 & NA & NA \\
\hline & M-202 & major constituents & 22 & $<0.02$ & 0.00 & NA & NA \\
\hline & PPREE-1 & trace constituents & 10 & 114 & 7.80 & NA & NA \\
\hline & SCREE-1 & trace constituents & 10 & 35.2 & 1.50 & NA & NA \\
\hline
\end{tabular}


Table 9. Analysis of quality control samples._Continued

[ $\mu \mathrm{g} / \mathrm{L}$, microgram per liter; mg/L, milligram per liter; NA, not applicable; $\mathrm{n}$, number; \%, percent; SD, standard deviation]

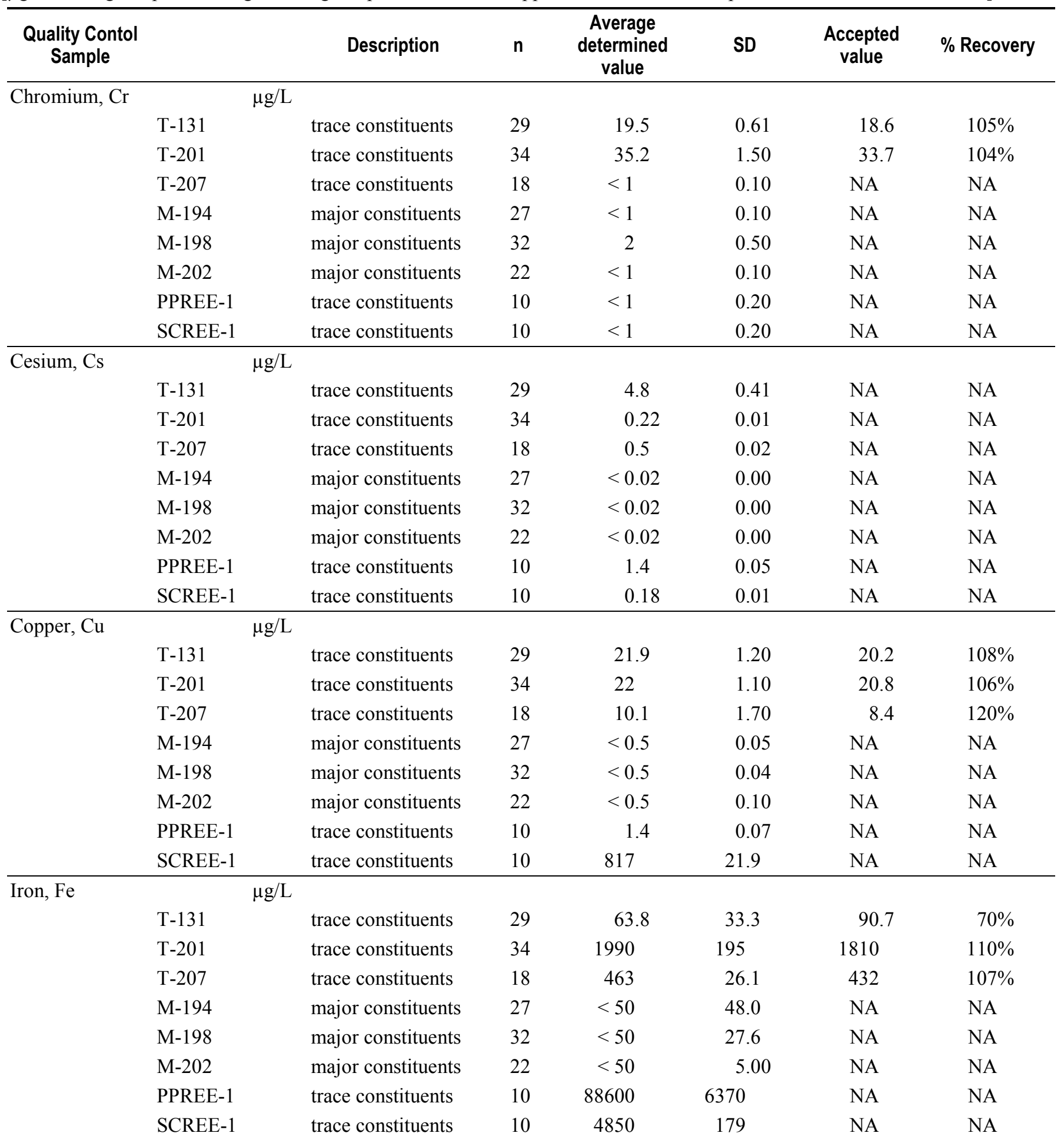


Table 9. Analysis of quality control samples.-Continued

[ $\mu \mathrm{g} / \mathrm{L}$, microgram per liter; mg/L, milligram per liter; NA, not applicable; $\mathrm{n}$, number; \%, percent; SD, standard deviation]

\begin{tabular}{|c|c|c|c|c|c|c|c|}
\hline $\begin{array}{l}\text { Quality Contol } \\
\text { Sample }\end{array}$ & & Description & $\mathrm{n}$ & $\begin{array}{c}\text { Average } \\
\text { determined } \\
\text { value }\end{array}$ & SD & $\begin{array}{l}\text { Accepted } \\
\text { value }\end{array}$ & $\%$ Recovery \\
\hline \multirow[t]{8}{*}{ Gallium, Ga } & & & & & & & \\
\hline & $\mathrm{T}-131$ & trace constituents & 29 & $<0.05$ & NA & NA & NA \\
\hline & $\mathrm{T}-201$ & trace constituents & 34 & 0.08 & 0.02 & NA & NA \\
\hline & $\mathrm{T}-207$ & trace constituents & 18 & 0.08 & 0.01 & NA & NA \\
\hline & M-198 & major constituents & 32 & $<0.05$ & NA & NA & NA \\
\hline & M-202 & major constituents & 22 & $<0.05$ & NA & NA & NA \\
\hline & PPREE-1 & trace constituents & 10 & 1.5 & 0.10 & NA & NA \\
\hline & SCREE-1 & trace constituents & 10 & 0.31 & 0.02 & NA & NA \\
\hline \multirow{6}{*}{ Gadolinium, Gd } & $\mathrm{T}-207$ & trace constituents & 18 & 0.27 & 0.03 & NA & NA \\
\hline & M-194 & major constituents & 27 & $<0.005$ & NA & NA & NA \\
\hline & M-198 & major constituents & 32 & $<0.005$ & NA & NA & NA \\
\hline & M-202 & major constituents & 22 & $<0.005$ & NA & NA & NA \\
\hline & PPREE-1 & trace constituents & 10 & 29.7 & 1.90 & 23.8 & $125 \%$ \\
\hline & SCREE-1 & trace constituents & 10 & 10.6 & 0.73 & 8.2 & $129 \%$ \\
\hline \multirow[t]{4}{*}{ Germanium, Ge } & & & & & & & \\
\hline & $\mathrm{T}-131$ & trace constituents & 29 & 0.1 & 0.00 & NA & NA \\
\hline & $\mathrm{T}-201$ & trace constituents & 34 & $<0.05$ & NA & NA & NA \\
\hline & SCREE-1 & trace constituents & 10 & 0.1 & 0.10 & 0.1 & $100 \%$ \\
\hline \multirow[t]{9}{*}{ Holmium, Ho } & & & & & & & \\
\hline & $\mathrm{T}-131$ & trace constituents & 29 & $<0.005$ & NA & NA & NA \\
\hline & $\mathrm{T}-201$ & trace constituents & 34 & 0.057 & 0.01 & NA & NA \\
\hline & $\mathrm{T}-207$ & trace constituents & 18 & 0.04 & 0.00 & NA & NA \\
\hline & M-194 & major constituents & 27 & $<0.005$ & NA & NA & NA \\
\hline & M-198 & major constituents & 32 & $<0.005$ & NA & NA & NA \\
\hline & M-202 & major constituents & 22 & $<0.005$ & NA & NA & NA \\
\hline & PPREE-1 & trace constituents & 10 & 5 & 0.40 & 4.4 & $114 \%$ \\
\hline & SCREE-1 & trace constituents & 10 & 1.9 & 0.19 & 1.6 & $119 \%$ \\
\hline
\end{tabular}


Table 9. Analysis of quality control samples.-Continued

[ $\mu \mathrm{g} / \mathrm{L}$, microgram per liter; mg/L, milligram per liter; NA, not applicable; $n$, number; \%, percent; SD, standard deviation]

\begin{tabular}{|c|c|c|c|c|c|c|c|}
\hline $\begin{array}{l}\text { Quality Contol } \\
\text { Sample }\end{array}$ & & Description & $\mathrm{n}$ & $\begin{array}{c}\text { Average } \\
\text { determined } \\
\text { value }\end{array}$ & SD & $\begin{array}{l}\text { Accepted } \\
\text { value }\end{array}$ & $\%$ Recovery \\
\hline \multirow[t]{8}{*}{ Potassium, K } & & & & & & & \\
\hline & $\mathrm{T}-131$ & trace constituents & 29 & 2.4 & 0.16 & 2.4 & $100 \%$ \\
\hline & $\mathrm{T}-201$ & trace constituents & 34 & 5.2 & 0.32 & 5.2 & $99 \%$ \\
\hline & $\mathrm{T}-207$ & trace constituents & 18 & 2.2 & 0.11 & 2.2 & $100 \%$ \\
\hline & M-198 & major constituents & 32 & 1.4 & 0.10 & 1.5 & $97 \%$ \\
\hline & M-202 & major constituents & 22 & 1.3 & 0.05 & 1.3 & $99 \%$ \\
\hline & PPREE-1 & trace constituents & 10 & 0.97 & 0.11 & NA & NA \\
\hline & SCREE-1 & trace constituents & 10 & 5.6 & 0.45 & NA & NA \\
\hline \multirow{6}{*}{ Lanthanum, La } & $\mathrm{T}-207$ & trace constituents & 18 & 1.7 & 0.08 & NA & NA \\
\hline & M-194 & major constituents & 27 & $<0.01$ & $<0.01$ & NA & NA \\
\hline & M-198 & major constituents & 32 & $<0.01$ & $<0.01$ & NA & NA \\
\hline & M-202 & major constituents & 22 & $<0.01$ & $<0.01$ & NA & NA \\
\hline & PPREE-1 & trace constituents & 10 & 86.4 & 3.61 & 80.4 & $110 \%$ \\
\hline & SCREE-1 & trace constituents & 10 & 10.6 & 0.44 & 9.8 & $110 \%$ \\
\hline \multirow[t]{4}{*}{ Lithium, Li } & & & & & & & \\
\hline & $\mathrm{T}-131$ & trace constituents & 29 & 16.4 & 1.67 & 17 & $100 \%$ \\
\hline & $\mathrm{T}-201$ & trace constituents & 34 & 27.3 & 1.60 & 27.9 & $100 \%$ \\
\hline & SCREE-1 & trace constituents & 10 & 9 & 2.61 & NA & NA \\
\hline \multirow[t]{9}{*}{ Lutetium, Lu } & & & & & & & \\
\hline & $\mathrm{T}-131$ & trace constituents & 29 & $<0.1$ & NA & NA & NA \\
\hline & $\mathrm{T}-201$ & trace constituents & 34 & $<0.1$ & NA & NA & NA \\
\hline & $\mathrm{T}-207$ & trace constituents & 18 & $<0.1$ & NA & NA & NA \\
\hline & M-194 & major constituents & 27 & $<0.1$ & NA & NA & NA \\
\hline & M-198 & major constituents & 32 & $<0.1$ & NA & NA & NA \\
\hline & M-202 & major constituents & 22 & $<0.1$ & NA & NA & NA \\
\hline & PPREE-1 & trace constituents & 10 & 1.3 & 0.05 & 1.1 & $110 \%$ \\
\hline & SCREE-1 & trace constituents & 10 & 0.5 & 0.04 & 0.5 & $110 \%$ \\
\hline
\end{tabular}


Table 9. Analysis of quality control samples.-Continued

[ $\mu \mathrm{g} / \mathrm{L}$, microgram per liter; mg/L, milligram per liter; NA, not applicable; $\mathrm{n}$, number; \%, percent; SD, standard deviation]

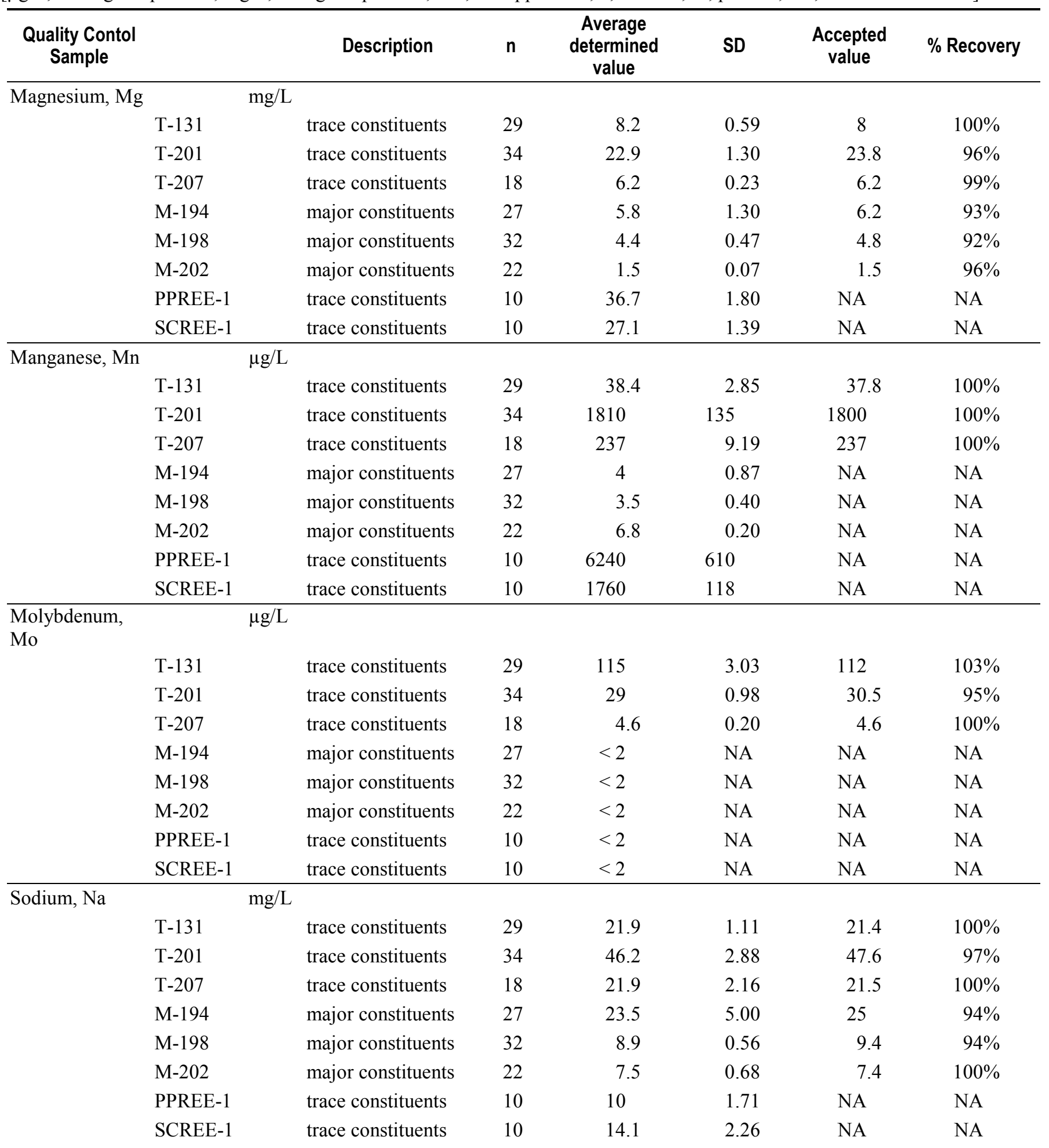


Table 9. Analysis of quality control samples.-Continued

[ $\mu \mathrm{g} / \mathrm{L}$, microgram per liter; mg/L, milligram per liter; NA, not applicable; n, number; \%, percent; SD, standard deviation]

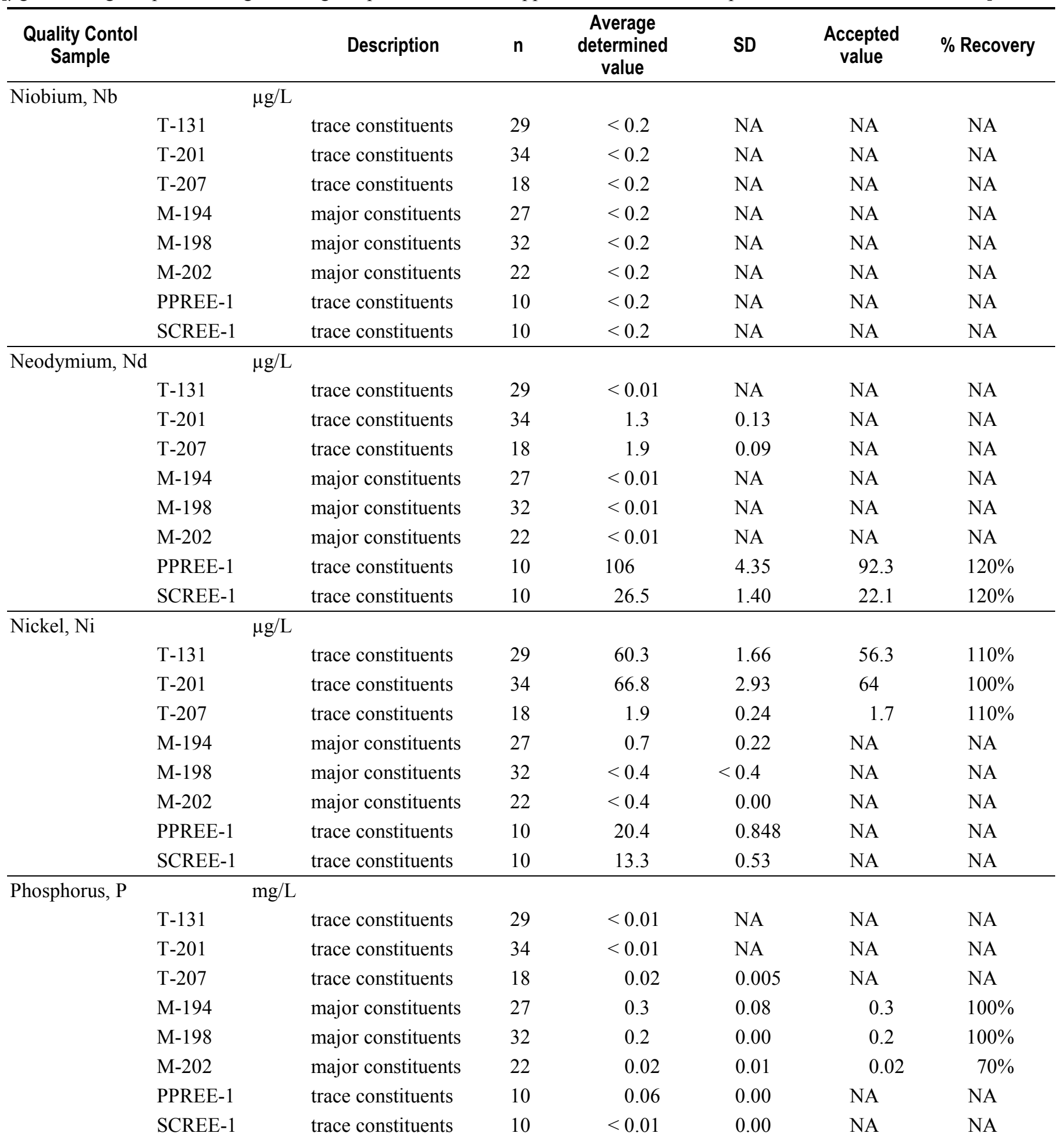


Table 9. Analysis of quality control samples.-Continued

[ $\mu \mathrm{g} / \mathrm{L}$, microgram per liter; mg/L, milligram per liter; NA, not applicable; $\mathrm{n}$, number; \%, percent; SD, standard deviation]

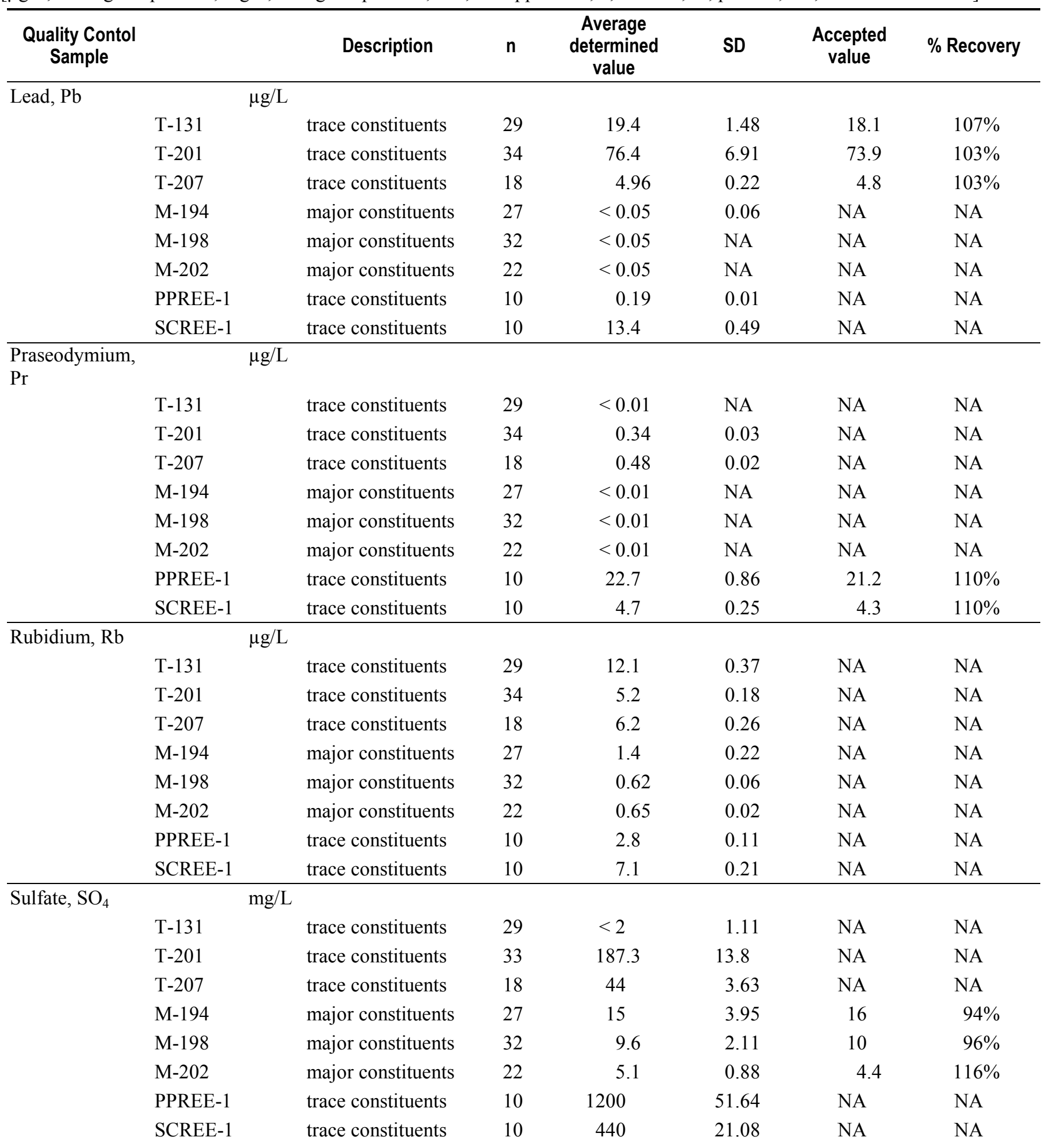


Table 9. Analysis of quality control samples.-Continued

[ $\mu \mathrm{g} / \mathrm{L}$, microgram per liter; mg/L, milligram per liter; NA, not applicable; n, number; \%, percent; SD, standard deviation]

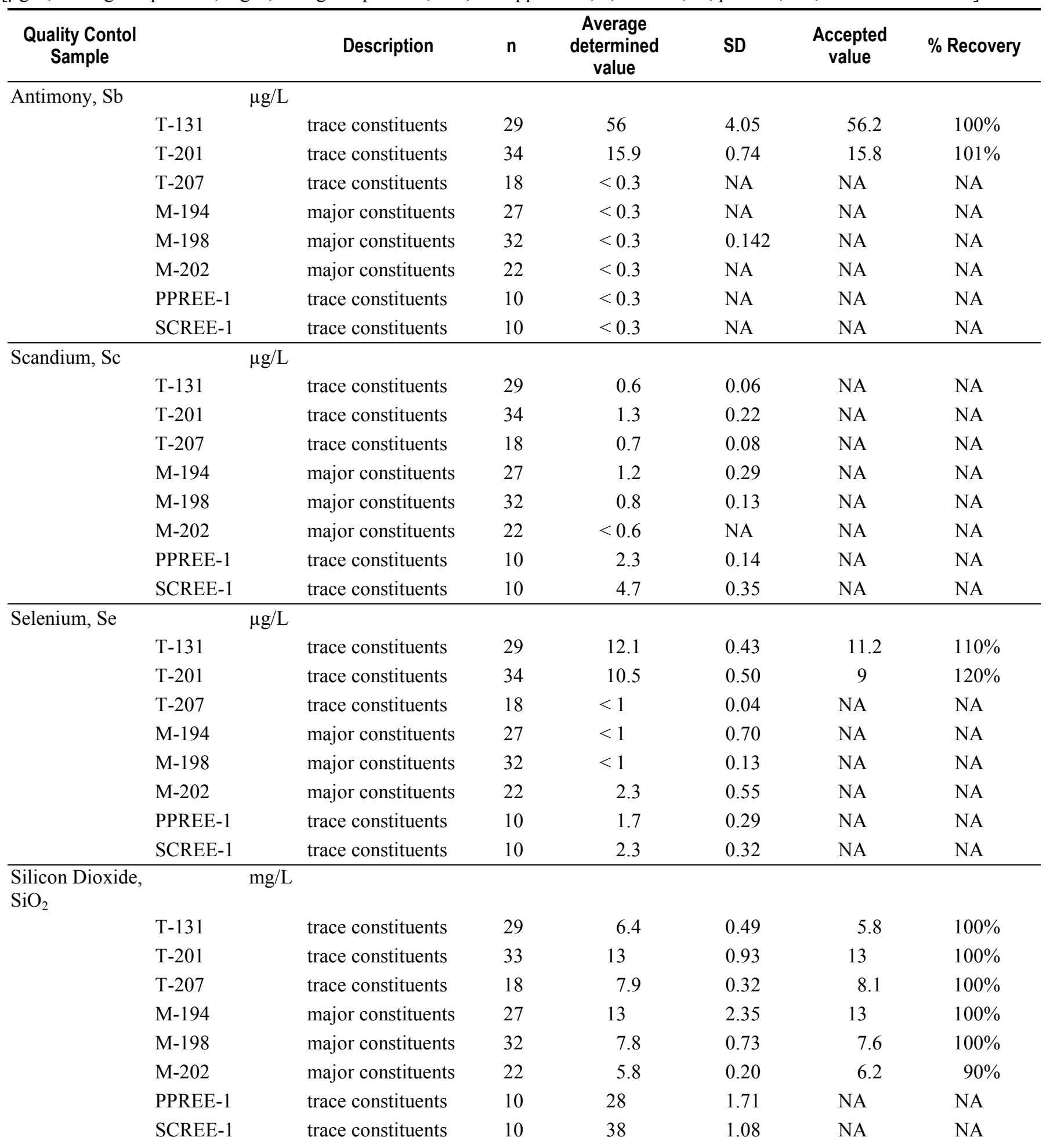


Table 9. Analysis of quality control samples.-Continued

[ $\mu \mathrm{g} / \mathrm{L}$, microgram per liter; mg/L, milligram per liter; NA, not applicable; $\mathrm{n}$, number; \%, percent; SD, standard deviation]

\begin{tabular}{|c|c|c|c|c|c|c|c|}
\hline $\begin{array}{l}\text { Quality Contol } \\
\text { Sample }\end{array}$ & & Description & $\mathrm{n}$ & $\begin{array}{c}\text { Average } \\
\text { determined } \\
\text { value }\end{array}$ & SD & $\begin{array}{l}\text { Accepted } \\
\text { value }\end{array}$ & \% Recovery \\
\hline \multirow[t]{8}{*}{ Samarium, Sm } & & & & & & & \\
\hline & $\mathrm{T}-131$ & trace constituents & 29 & $<0.01$ & NA & NA & NA \\
\hline & $\mathrm{T}-201$ & trace constituents & 33 & 0.27 & 0.03 & NA & NA \\
\hline & $\mathrm{T}-207$ & trace constituents & 18 & 0.34 & 0.02 & NA & NA \\
\hline & M-198 & major constituents & 32 & $<0.01$ & NA & NA & NA \\
\hline & M-202 & major constituents & 22 & $<0.01$ & NA & NA & NA \\
\hline & PPREE-1 & trace constituents & 10 & 23.1 & 0.95 & 20.3 & $110 \%$ \\
\hline & SCREE-1 & trace constituents & 10 & 8 & 0.52 & 6.7 & $120 \%$ \\
\hline \multirow{6}{*}{ Strontium, $\mathrm{Sr}$} & $\mathrm{T}-207$ & trace constituents & 18 & 157 & 5.87 & 161 & $97 \%$ \\
\hline & M-194 & major constituents & 25 & 129 & 13 & 135 & $95 \%$ \\
\hline & M-198 & major constituents & 32 & 47.9 & 4.28 & 52.4 & $91 \%$ \\
\hline & M-202 & major constituents & 22 & 36 & 1.32 & 37.4 & $96 \%$ \\
\hline & PPREE-1 & trace constituents & 10 & 2850 & 128 & NA & NA \\
\hline & SCREE-1 & trace constituents & 10 & 123 & 3.93 & NA & NA \\
\hline \multirow[t]{4}{*}{ Tantalum, Ta } & & & & & & & \\
\hline & $\mathrm{T}-131$ & trace constituents & 29 & $<0.02$ & 0.01 & NA & NA \\
\hline & $\mathrm{T}-201$ & trace constituents & 33 & $<0.02$ & 0.01 & NA & NA \\
\hline & SCREE-1 & trace constituents & 10 & $<0.02$ & NA & NA & NA \\
\hline \multirow[t]{9}{*}{ Terbium, Tb } & & & & & & & \\
\hline & $\mathrm{T}-131$ & trace constituents & 29 & $<0.005$ & NA & NA & NA \\
\hline & $\mathrm{T}-201$ & trace constituents & 33 & 0.05 & 0.01 & NA & NA \\
\hline & $\mathrm{T}-207$ & trace constituents & 18 & 0.04 & 0.00 & NA & NA \\
\hline & M-194 & major constituents & 27 & $<0.005$ & NA & NA & NA \\
\hline & M-198 & major constituents & 32 & $<0.005$ & NA & NA & NA \\
\hline & M-202 & major constituents & 22 & $<0.005$ & NA & NA & NA \\
\hline & PPREE-1 & trace constituents & 10 & 4 & 0.28 & 3.6 & $110 \%$ \\
\hline & SCREE-1 & trace constituents & 10 & 1.5 & 0.12 & 1.3 & $110 \%$ \\
\hline
\end{tabular}


Table 9. Analysis of quality control samples.-Continued

[ $\mu \mathrm{g} / \mathrm{L}$, microgram per liter; mg/L, milligram per liter; NA, not applicable; $\mathrm{n}$, number; \%, percent; SD, standard deviation]

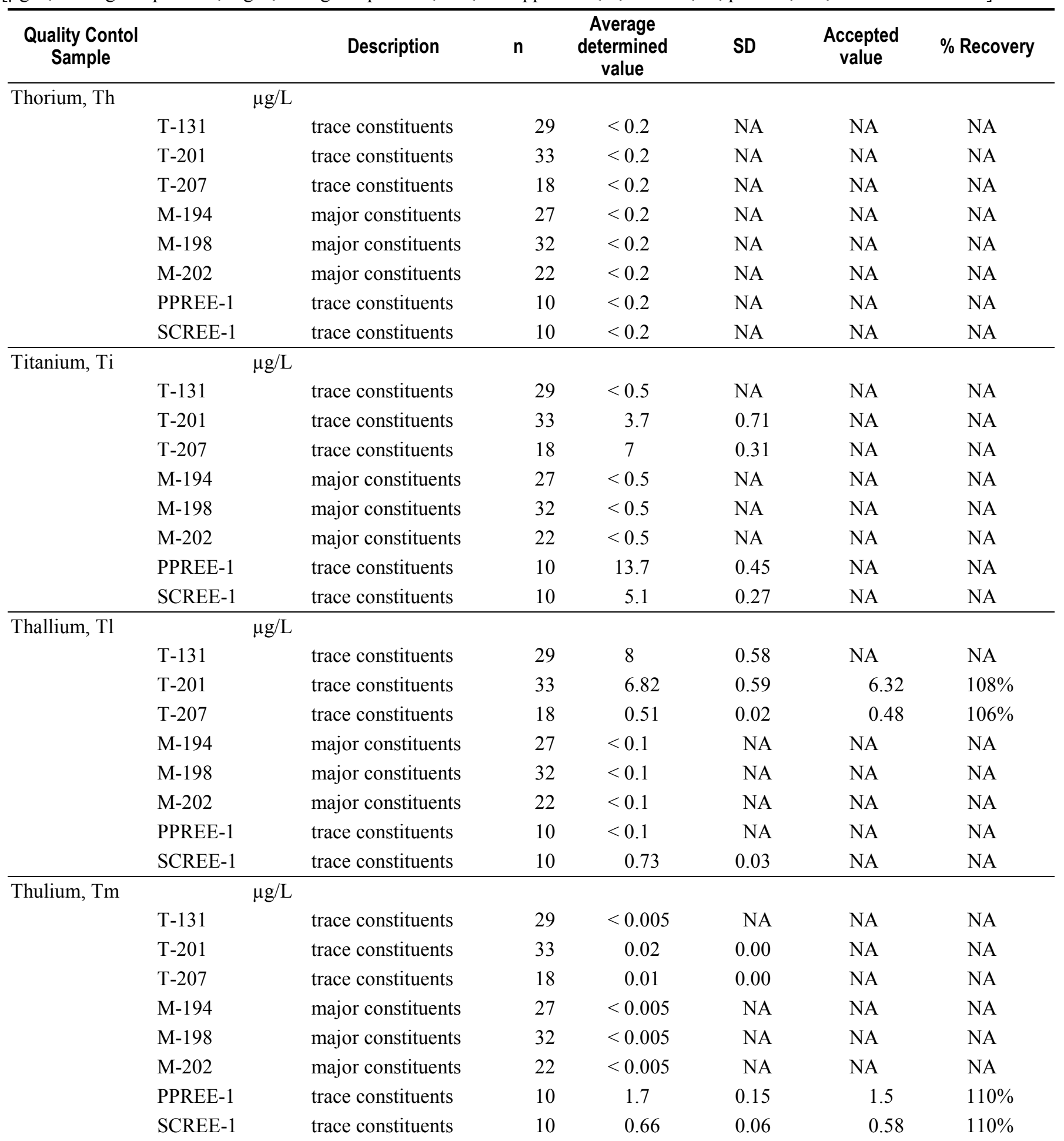


Table 9. Analysis of quality control samples.-Continued

$[\mu \mathrm{g} / \mathrm{L}$, microgram per liter; mg/L, milligram per liter; NA, not applicable; $\mathrm{n}$, number; \%, percent; SD, standard deviation]

\begin{tabular}{|c|c|c|c|c|c|c|c|}
\hline $\begin{array}{l}\text { Quality Contol } \\
\text { Sample }\end{array}$ & & Description & $\mathrm{n}$ & $\begin{array}{c}\text { Average } \\
\text { determined } \\
\text { value }\end{array}$ & SD & $\begin{array}{l}\text { Accepted } \\
\text { value }\end{array}$ & \% Recovery \\
\hline \multirow[t]{9}{*}{ Uranium, U } & & & & & & & \\
\hline & $\mathrm{T}-131$ & trace constituents & 29 & $<0.1$ & NA & NA & NA \\
\hline & $\mathrm{T}-201$ & trace constituents & 33 & 9.56 & 1.11 & 9.22 & $104 \%$ \\
\hline & $\mathrm{T}-207$ & trace constituents & 18 & 1.87 & 0.10 & 1.8 & $104 \%$ \\
\hline & M-194 & major constituents & 27 & 1.65 & 0.47 & NA & NA \\
\hline & M-198 & major constituents & 32 & 0.2 & 0.04 & NA & NA \\
\hline & M-202 & major constituents & 22 & 0.17 & 0.01 & NA & NA \\
\hline & PPREE-1 & trace constituents & 10 & 0.13 & 0.007 & NA & NA \\
\hline & SCREE-1 & trace constituents & 10 & 0.28 & 0.02 & NA & NA \\
\hline \multirow[t]{9}{*}{ Vanadium, V } & & & & & & & \\
\hline & $\mathrm{T}-131$ & trace constituents & 29 & 36.8 & 1.18 & 34.2 & $110 \%$ \\
\hline & $\mathrm{T}-201$ & trace constituents & 33 & 29.2 & 1.33 & 28.4 & $100 \%$ \\
\hline & $\mathrm{T}-207$ & trace constituents & 18 & 0.9 & 0.06 & NA & NA \\
\hline & M-194 & major constituents & 27 & 2.7 & 0.18 & 2.8 & $100 \%$ \\
\hline & M-198 & major constituents & 32 & 6.4 & 0.41 & 6.6 & $100 \%$ \\
\hline & M-202 & major constituents & 22 & $<0.5$ & NA & NA & NA \\
\hline & PPREE-1 & trace constituents & 10 & 1.8 & 0.25 & NA & NA \\
\hline & SCREE-1 & trace constituents & 10 & $<0.5$ & NA & NA & NA \\
\hline \multirow[t]{9}{*}{ Tungsten, W } & & & & & & & \\
\hline & $\mathrm{T}-131$ & trace constituents & 29 & $<0.5$ & NA & NA & NA \\
\hline & $\mathrm{T}-201$ & trace constituents & 33 & $<0.5$ & NA & NA & NA \\
\hline & $\mathrm{T}-207$ & trace constituents & 18 & $<0.5$ & NA & NA & NA \\
\hline & M-194 & major constituents & 27 & $<0.5$ & NA & NA & NA \\
\hline & M-198 & major constituents & 32 & $<0.5$ & 0.54 & NA & NA \\
\hline & M-202 & major constituents & 22 & $<0.5$ & NA & NA & NA \\
\hline & PPREE-1 & trace constituents & 10 & $<0.5$ & NA & NA & NA \\
\hline & SCREE-1 & trace constituents & 10 & $<0.5$ & NA & NA & NA \\
\hline \multirow[t]{9}{*}{ Yttrium, Y } & & & & & & & \\
\hline & $\mathrm{T}-131$ & trace constituents & 29 & $<0.01$ & NA & NA & NA \\
\hline & $\mathrm{T}-201$ & trace constituents & 33 & 2 & 0.07 & NA & NA \\
\hline & $\mathrm{T}-207$ & trace constituents & 18 & 1 & 0.05 & NA & NA \\
\hline & M-194 & major constituents & 27 & $<0.01$ & NA & NA & NA \\
\hline & M-198 & major constituents & 32 & $<0.01$ & NA & NA & NA \\
\hline & M-202 & major constituents & 22 & $<0.01$ & NA & NA & NA \\
\hline & PPREE-1 & trace constituents & 10 & 140 & 0.279 & NA & NA \\
\hline & SCREE-1 & trace constituents & 10 & 45.1 & 1.40 & NA & NA \\
\hline
\end{tabular}


Table 9. Analysis of quality control samples.-Continued

[ $\mu \mathrm{g} / \mathrm{L}$, microgram per liter; mg/L, milligram per liter; NA, not applicable; $\mathrm{n}$, number; \%, percent; SD, standard deviation]

\begin{tabular}{|c|c|c|c|c|c|c|c|}
\hline $\begin{array}{l}\text { Quality Contol } \\
\text { Sample }\end{array}$ & & Description & $\mathrm{n}$ & $\begin{array}{c}\text { Average } \\
\text { determined } \\
\text { value }\end{array}$ & SD & $\begin{array}{l}\text { Accepted } \\
\text { value }\end{array}$ & $\%$ Recovery \\
\hline \multirow[t]{9}{*}{ Ytterbium, $\mathrm{Yb}$} & & & & & & & \\
\hline & $\mathrm{T}-131$ & trace constituents & 29 & $<0.01$ & NA & NA & NA \\
\hline & $\mathrm{T}-201$ & trace constituents & 33 & 0.1 & 0.01 & NA & NA \\
\hline & $\mathrm{T}-207$ & trace constituents & 18 & 0.07 & 0.01 & NA & NA \\
\hline & M-194 & major constituents & 27 & $<0.01$ & NA & NA & NA \\
\hline & M-198 & major constituents & 32 & $<0.01$ & NA & NA & NA \\
\hline & M-202 & major constituents & 22 & $<0.01$ & NA & NA & NA \\
\hline & PPREE-1 & trace constituents & 10 & 9.5 & 0.59 & NA & NA \\
\hline & SCREE-1 & trace constituents & 10 & 4 & 0.26 & NA & NA \\
\hline \multirow[t]{9}{*}{ Zinc, $\mathrm{Zn}$} & & & & & & & \\
\hline & $\mathrm{T}-131$ & trace constituents & 29 & 75 & 3.26 & 72 & $104 \%$ \\
\hline & $\mathrm{T}-201$ & trace constituents & 33 & 1200 & 54 & 1110 & $108 \%$ \\
\hline & $\mathrm{T}-207$ & trace constituents & 18 & 225 & 7.75 & 200 & $113 \%$ \\
\hline & M-194 & major constituents & 27 & 4.3 & 0.58 & NA & NA \\
\hline & M-198 & major constituents & 32 & $<3$ & NA & NA & NA \\
\hline & M-202 & major constituents & 22 & $<3$ & 0.06 & NA & NA \\
\hline & PPREE-1 & trace constituents & 10 & 586 & 34 & NA & NA \\
\hline & SCREE-1 & trace constituents & 10 & 2030 & 72 & NA & NA \\
\hline \multirow[t]{9}{*}{ Zirconium, Zr } & & & & & & & \\
\hline & $\mathrm{T}-131$ & trace constituents & 29 & $<0.2$ & NA & NA & NA \\
\hline & $\mathrm{T}-201$ & trace constituents & 33 & $<0.2$ & 0.08 & NA & NA \\
\hline & $\mathrm{T}-207$ & trace constituents & 18 & $<0.2$ & 0.06 & NA & NA \\
\hline & M-194 & major constituents & 27 & $<0.2$ & NA & NA & NA \\
\hline & M-198 & major constituents & 32 & $<0.2$ & NA & NA & NA \\
\hline & M-202 & major constituents & 22 & $<0.2$ & NA & NA & NA \\
\hline & PPREE-1 & trace constituents & 10 & $<0.2$ & NA & NA & NA \\
\hline & SCREE-1 & trace constituents & 10 & $<0.2$ & NA & NA & NA \\
\hline
\end{tabular}

\section{References Cited}

Abou-Shakar, F., 2005, Extending the dynamic range of the ELAN DRC by selective attenuation of high signals: Shelton, Conn., PerkinElmer, Inc., Field Application Report \#007437_01, 3 p., accessed July 7, 2014, at http://perkinelmer.co.kr/files/AP00155.pdf.

Allain, Pierre, Jaunalt, Laurent, Mauras, Yves, Mermet, Jean Michel, and Delaporte, Thierry, 1991, Signal enhancement of elements due to the presence of carbon-containing compounds in inductively coupled plasma mass spectrometry: Analytical Chemistry, v. 63, no. 14, p. 1497-1498.

Horlick, Gary, and Montaser, Akbar, 1998, Analytical characteristics of ICP-MS in inductively coupled plasma mass spectrometry (Montaser, Akbar, ed.): New York, New York, United States, Wiley-VCH, Inc., ISBN 0-0471-18620-1, p. 503-588. 
Jarvis, K.E., Gray, A.L., and Houk, R.S., 1992, Handbook of Inductively Coupled Plasma Mass Spectrometry: Glasgow, Scotland, United Kingdom, Blackie and Son Ltd., ISBN 0-216-92912-1, p $125-152$.

Kralj, Polonca, and Veber, Marjan, 2003, Investigations into nonspectroscopic effects of organic compounds inductively coupled plasma mass spectrometry: Acta Chimica Slovenica, v. 50, p. 633644.

Lichte, F.E., Meier, A.L., and Crock, J.G., 1987, Determination of the rare earth elements in geological materials by inductively coupled plasma mass spectrometry: Analytical Chemistry, v. 59, no. 8, p. $1150-1157$.

Olesik, J.W., and Bates, L.C., 1995, Characterization of aerosols produced by pneumatic nebulizers for inductively coupled plasma sample introduction-Effect of liquid and gas flow rates on volume based drop size distributions: Spectrochimica Acta, v. 50B, nos. 4-7, p. 285-303.

Tanner, S.D., and Baranov, V.I., 1999, A dynamic reaction cell for inductively coupled plasma mass spectrometry (ICPDRC-MS)—Part II—Reduction of interferences produced within the cell: Journal of the American Society of Mass Spectrometry, v. 10, p. 1083-1094.

Tanner, S.D., Baranov, V.I., and Bandura, D.R., 2002, Reaction cells and collision cells for ICP-MSA tutorial review: Spectrochimica Acta Part B, v. 57, p. 1361-1452.

U.S. Environmental Protection Agency, 2007a, SW-846-Test methods for evaluating solid waste, physical/chemical methods, Method 3015A, microwave assisted acid digestion of aqueous samples and extracts: U.S. Environmental Protection Agency, revision 1, February 2007, accessed June 25, 2014, at http:/www.epa.gov/osw/hazard/testmethods/sw846/pdfs/3015a.pdf.

U.S. Environmental Protection Agency, 2007b, SW-846-Test methods for evaluating solid waste, physical/chemical methods, Method 6020A, inductively coupled plasma mass spectrometry: U.S. Environmental Protection Agency, revision 1, February 2007, accessed July 7, 2014, at http://www.epa.gov/osw/hazard/testmethods/sw846/pdfs/6020a.pdf.

U.S. Geological Survey, 2006, Collection of water samples (ver. 2.0): U.S. Geological Survey Techniques of Water-Resources Investigations, book 9, chap. A4, September 2006, accessed January 6, 2014, at http://pubs.water.usgs.gov/twri9A4/.

Verplanck, P. L., Antweiler, R. C., Nordstrom, D. K., Taylor, H. E., 2001, Standard reference water samples for rare earth element determinations: Applied Geochemistry, v. 16, p. 231-244.

Wanty, R.B., Wang, Bronwen, Vohden, Jim, Briggs, P.H., and Meier, A.H., 1999, Regional baseline geochemistry and environmental effects of gold placer mining operations on the Fortymile River, eastern Alaska: U.S. Geological Survey Open-File Report 99-328, p. 11.

Wilde, F.D., Radtke, D.B., Gibs, Jacob, and Iwatsubo, R.T., eds., 2004-2009, Processing of water samples (ver. 2.2): U.S. Geological Survey Techniques of Water-Resources Investigations, book 9 , chap. A5, April 2004, accessed July 7, 2014, at http://pubs.water.usgs.gov/twri9A5/.

Wolf, R.E., Denoyer, Eric, and Grosser, Zoe, 2001, U.S. EPA Method 200.8 for the analysis of drinking waters and wastewaters: PerkinElmer Instruments, ICP-MS Application Note D-6527, Second Edition, accessed July 7, 2014, at http://perkinelmer.co.kr/files/AP00031.pdf. 


\section{Appendix 1. Determination of Empirical Oxide Corrections}

For the rare earth elements (REEs), elements affected by high levels of $\mathrm{Ca}$ in samples such as $\mathrm{Fe}, \mathrm{Ni}$, and $\mathrm{Co}$, and for the correction of ${ }^{91} \mathrm{Zr}^{16} \mathrm{O}$ and ${ }^{93} \mathrm{Nb}^{16} \mathrm{O}$ interferences on ${ }^{107} \mathrm{Ag}$ and ${ }^{109} \mathrm{Ag}$, respectively; empirical oxide correction equations are used that must be reevaluated on a routine basis as instrument tuning parameters are changed. Before performing oxide corrections, optimize the instrument and record the $\mathrm{CeO} / \mathrm{Ce}$ ratio from the Daily Performance Report. If operating parameters result in a $\mathrm{CeO} / \mathrm{Ce}$ ratio more than 50 percent different than when the corrections were previously evaluated, the oxide corrections should be redetermined. The instrumental parameters for determining oxide corrections are given in table $1-1$.

Table 1-1. Elements and timing parameters for oxide interference correction determination.

[cps, counts per second; MCA, multichannel analyzer; ms, milliseconds]

\begin{tabular}{|c|c|c|c|c|c|}
\hline \multicolumn{6}{|c|}{$\begin{array}{l}\text { Number of Sweeps: } 15 \\
\text { Number of Replicates: } 3\end{array}$} \\
\hline Element & Mass & Scan mode & $\begin{array}{c}\text { MCA } \\
\text { channels }\end{array}$ & $\begin{array}{l}\text { Dwell time } \\
\text { (ms) }\end{array}$ & $\begin{array}{c}\text { Integration } \\
\text { time (ms) }\end{array}$ \\
\hline $\mathrm{Ca}$ & 42.9588 & Peak Hopping & 1 & 50 & 750 \\
\hline $\mathrm{Ca}$ & 43.9555 & Peak Hopping & 1 & 50 & 750 \\
\hline $\mathrm{Fe}$ & 56.9354 & Peak Hopping & 1 & 50 & 750 \\
\hline $\mathrm{Ni}$ & 57.9353 & Peak Hopping & 1 & 50 & 750 \\
\hline Co & 58.9332 & Peak Hopping & 1 & 50 & 750 \\
\hline $\mathrm{Ni}$ & 59.9332 & Peak Hopping & 1 & 50 & 750 \\
\hline $\mathrm{Ni}$ & 60.931 & Peak Hopping & 1 & 50 & 750 \\
\hline $\mathrm{Zr}$ & 89.9043 & Peak Hopping & 1 & 50 & 750 \\
\hline $\mathrm{Nb}$ & 92.906 & Peak Hopping & 1 & 50 & 750 \\
\hline Mo & 94.9058 & Peak Hopping & 1 & 50 & 750 \\
\hline Mo & 97.9055 & Peak Hopping & 1 & 50 & 750 \\
\hline $\mathrm{Ag}$ & 106.905 & Peak Hopping & 1 & 50 & 750 \\
\hline $\mathrm{Ag}$ & 108.905 & Peak Hopping & 1 & 50 & 750 \\
\hline $\mathrm{Cd}$ & 110.904 & Peak Hopping & 1 & 50 & 750 \\
\hline $\mathrm{Cd}$ & 113.904 & Peak Hopping & 1 & 50 & 750 \\
\hline $\mathrm{Ba}$ & 134.906 & Peak Hopping & 1 & 50 & 750 \\
\hline $\mathrm{Ba}$ & 137.905 & Peak Hopping & 1 & 50 & 750 \\
\hline $\mathrm{La}$ & 138.906 & Peak Hopping & 1 & 50 & 750 \\
\hline $\mathrm{Ce}$ & 139.905 & Peak Hopping & 1 & 50 & 750 \\
\hline $\operatorname{Pr}$ & 140.907 & Peak Hopping & 1 & 50 & 750 \\
\hline $\mathrm{Nd}$ & 142.91 & Peak Hopping & 1 & 50 & 750 \\
\hline $\mathrm{Nd}$ & 145.913 & Peak Hopping & 1 & 50 & 750 \\
\hline $\mathrm{Sm}$ & 146.915 & Peak Hopping & 1 & 50 & 750 \\
\hline $\mathrm{Sm}$ & 148.917 & Peak Hopping & 1 & 50 & 750 \\
\hline $\mathrm{Eu}$ & 150.92 & Peak Hopping & 1 & 50 & 750 \\
\hline $\mathrm{Eu}$ & 152.929 & Peak Hopping & 1 & 50 & 750 \\
\hline
\end{tabular}


Table 1-1. Elements and timing parameters for oxide interference correction determination.

[cps, counts per second; MCA, multichannel analyzer; ms, milliseconds]

\begin{tabular}{lccccc}
\hline \multicolumn{2}{l}{$\begin{array}{l}\text { Number of Sweeps: } \mathbf{1 5} \\
\text { Number of Replicates: } \mathbf{3}\end{array}$} & \multicolumn{3}{l}{} \\
\hline Element & Mass & Scan mode & $\begin{array}{c}\text { MCA } \\
\text { channels }\end{array}$ & $\begin{array}{c}\text { Dwell time } \\
\text { (ms) }\end{array}$ & $\begin{array}{c}\text { Integration } \\
\text { time (ms) }\end{array}$ \\
\hline Gd & 157.924 & Peak Hopping & 1 & 50 & 750 \\
$\mathrm{~Tb}$ & 158.925 & Peak Hopping & 1 & 50 & 750 \\
$\mathrm{Gd}$ & 159.927 & Peak Hopping & 1 & 50 & 750 \\
$\mathrm{Dy}$ & 160.927 & Peak Hopping & 1 & 50 & 750 \\
$\mathrm{Dy}$ & 163.929 & Peak Hopping & 1 & 50 & 750 \\
$\mathrm{Ho}$ & 164.93 & Peak Hopping & 1 & 50 & 750 \\
$\mathrm{Er}$ & 165.93 & Peak Hopping & 1 & 50 & 750 \\
$\mathrm{Tm}$ & 168.934 & Peak Hopping & 1 & 50 & 750 \\
$\mathrm{Yb}$ & 171.937 & Peak Hopping & 1 & 50 & 750 \\
$\mathrm{Yb}$ & 172.938 & Peak Hopping & 1 & 50 & 750 \\
$\mathrm{Lu}$ & 174.941 & Peak Hopping & 1 & 50 & 750 \\
$\mathrm{Hf}$ & 178.946 & Peak Hopping & 1 & 50 & 750 \\
$\mathrm{Hf}$ & 179.947 & Peak Hopping & 1 & 50 & 750 \\
$\mathrm{Ta}$ & 180.948 & Peak Hopping & 1 & 50 & 750 \\
$\mathrm{~W}$ & 183.951 & Peak Hopping & 1 & 50 & 750 \\
\hline
\end{tabular}

\section{Procedure for Determining Oxide Corrections}

1. Create a quantitative method using the isotopes and timing parameters given in table $1-1$. Be sure to go into the Equations tab in the Method Editor and delete ALL correction equations. Save the method under a suitable name such as Oxide Corrections.mth. The resulting data can either be printed or sent to an electronic file.

2. Prepare single element solutions of the following elements at a concentration of 10 micrograms per liter $(\mu \mathrm{g} / \mathrm{L})-\mathrm{Zr}, \mathrm{Nb}, \mathrm{Mo}, \mathrm{Ba}, \mathrm{La}, \mathrm{Ce}, \mathrm{Pr}, \mathrm{Nd}, \mathrm{Sm}, \mathrm{Eu}, \mathrm{Gd}, \mathrm{Tb}, \mathrm{Dy}, \mathrm{Ho}, \mathrm{Er}$, $\mathrm{Tm}, \mathrm{Yb}, \mathrm{Lu}, \mathrm{Hf}$, Ta, and W; A 50 milliliter $(\mathrm{mL})$ volume of each is sufficient for several analyses.

3. Prepare a single element solution of calcium (Ca) at 10 milligram per liter $(\mathrm{mg} / \mathrm{L})$; a $50 \mathrm{~mL}$ volume is sufficient for several analyses.

4. Prepare $50 \mathrm{~mL}$ of 2 percent nitric acid $\left(\mathrm{HNO}_{3}\right)$ using double-distilled nitric acid and 18 megaohm $(\mathrm{M} \Omega)$ deionized water to be used as a blank solution.

5. Set up the autosampler to run a minimum of 5 blanks initially, followed by a single analysis of each individual element solution with two blanks in between each solution. A washout time of at least two minutes between each solution is recommended to prevent carryover effects.

6. Once the solutions are analyzed, enter the resulting intensities for the blanks and each individual element solution into the shaded areas of the oxide correction calculations spreadsheet in table 1-2. The basis for calculating the correction equations is to calculate the ratio of the apparent signal for each interfering element at the interfered with element's mass 
to the net intensity of the interfering element. This correction ratio is then multiplied by the measured intensity of each interfering element measured in the sample solutions to adjust for changing concentrations of the interfering elements. Whenever possible, choose masses to measure the interfering element signals that do not contain interferences. The equations used to perform calculations are shown in table 1-2. All formulas for table 1-2 are available in a downloadable Microsoft Excel file at http://pubs.usgs.gov/of/2015/1010/pdf/ofr20151010_table1-2.xlsx. Collected data are entered into the appropriate blank fields and the calculations are performed automatically.

7. The spreadsheet in table 1-2 contains sections for elements where there are multiple interfering elements affecting an analyte mass. The correction equations for the first section, called First Order Corrections, for the REEs are calculated and then entered into the analytical method used to collect the data and the raw data reprocessed for the elements contained in the next set of REE correction equations, called Second Order Corrections in table 1-2. The resulting corrected raw data are then entered into the second area of the spreadsheet to generate the Second Order Corrections. The second order corrections are then entered into the analytical method in addition to any first order corrections and the data reprocessed again to generate the corrected raw data for generation of the Third Order Corrections in the third area of the spreadsheet. At each step, the analyst will review the results for the corrected raw data to ensure the corrections are resulting in signals for the interference-affected elements near the background levels observed in the blank solution analyses.

8. The corrections in the Other Oxide corrections area of the spreadsheet in table 1-2 can be generated along with the First Order Corrections, because these are not interrelated to the rare earth element oxide corrections.

9. The correction equations generated in the spreadsheet in table 1-2 must be saved, recorded, and entered into every individual method where these correction equations should be utilized. Note that the ELAN Instrument Control software offers no capability for correction equations to be automatically updated in multiple methods. 
Table 1-2. Correction equations calculation worksheet with formulas used for calculations shown.

[cps, counts per second]

\begin{tabular}{|c|c|c|c|c|c|c|c|c|c|c|c|c|c|c|c|c|}
\hline & $\mathbf{A}$ & $\mathbf{B}$ & $\mathbf{C}$ & $\mathbf{D}$ & $\mathbf{E}$ & $\mathbf{F}$ & $\mathbf{G}$ & $\mathbf{H}$ & I & $\mathbf{J}$ & $\mathbf{K}$ & $\mathbf{L}$ & $\mathbf{M}$ & $\mathbf{M}$ & $\mathbf{0}$ & $\mathbf{P}$ \\
\hline 1 & $\begin{array}{c}\text { First Order } \\
\text { Corrections } \\
\text { interfering } \\
\text { element }\end{array}$ & $\begin{array}{l}\text { Blank } \\
\text { (cps) }\end{array}$ & $\begin{array}{l}\text { Blank } \\
\text { (cps) }\end{array}$ & $\begin{array}{l}\text { Average blank } \\
\text { (cps) }\end{array}$ & $\begin{array}{c}\mathrm{Hi} \\
\text { standard } \\
\text { (cps) }\end{array}$ & $\begin{array}{c}\text { Net } \\
\text { intensity } \\
\text { for } \\
\text { element }\end{array}$ & $\begin{array}{l}\text { Interfered } \\
\text { with } \\
\text { element }\end{array}$ & $\begin{array}{l}\text { Blank } \\
\text { (cps) }\end{array}$ & $\begin{array}{l}\text { Blank } \\
\text { (cps) }\end{array}$ & $\begin{array}{l}\text { Average blank } \\
\text { (cps) }\end{array}$ & $\begin{array}{l}\text { Apparent } \\
\text { Element } \\
\text { Intensity } \\
\text { (cps) }\end{array}$ & $\begin{array}{c}\text { Net } \\
\text { apparent } \\
\text { element } \\
\text { intensity } \\
\text { (cps) }\end{array}$ & $\begin{array}{l}\text { Calculated } \\
\text { correction }\end{array}$ & $\begin{array}{l}\text { Interfering } \\
\text { element }\end{array}$ & \multicolumn{2}{|c|}{ Correction to enter } \\
\hline 2 & Ba135 & & & $=$ AVERAGE $(\mathrm{B} 2: \mathrm{C} 2)$ & & $=\mathrm{E} 2-\mathrm{D} 2$ & Eu151 & & & $=$ AVERAGE $(\mathrm{H} 2: \mathrm{I} 2)$ & & $=\mathrm{K} 2-\mathrm{J} 2$ & $=\mathrm{L} 2 / \mathrm{F} 2$ & $=\mathrm{G} 2$ & $=-1 * \mathrm{M} 2$ & $=" * " \& A 2$ \\
\hline 3 & Ba135 & & & $=$ AVERAGE(B3:C3) & & $=\mathrm{E} 3-\mathrm{D} 3$ & Nd146 & & & $=$ AVERAGE $(\mathrm{H} 3: \mathrm{I} 3)$ & & $=\mathrm{K} 3-\mathrm{J} 3$ & $=\mathrm{L} 3 / \mathrm{F} 3$ & $=\mathrm{G} 3$ & $=-1 * \mathrm{M} 3$ & $=" * " \& \mathrm{~A} 3$ \\
\hline 4 & $\mathrm{Nd} 146$ & & & $=\operatorname{AVERAGE}(\mathrm{B} 4: \mathrm{C} 4)$ & & $=$ E4-D4 & Tb159 & & & $=\operatorname{AVERAGE}(\mathrm{H} 4: \mathrm{I} 4)$ & & $=\mathrm{K} 4-\mathrm{J} 4$ & $=\mathrm{L} 4 / \mathrm{F} 4$ & $=\mathrm{G} 4$ & $=-1 * \mathrm{M} 4$ & $=" * " \& A 4$ \\
\hline 5 & $\operatorname{Sm} 149$ & & & $=$ AVERAGE $(B 5: C 5)$ & & $=\mathrm{E} 5-\mathrm{D} 5$ & Ho165 & & & $=\operatorname{AVERAGE}(\mathrm{H} 5: \mathrm{I5})$ & & $=\mathrm{K} 5-\mathrm{J} 5$ & $=\mathrm{L} 5 / \mathrm{F} 5$ & $=\mathrm{G} 5$ & $=-1 * \mathrm{M} 5$ & $=" * " \& \mathrm{~A} 5$ \\
\hline 6 & Eu151 & & & $=$ AVERAGE $(\mathrm{B} 6: \mathrm{C} 6)$ & & $=\mathrm{E} 6-\mathrm{D} 6$ & $\operatorname{Tm} 169$ & & & $=$ AVERAGE(H6:I6) & & $=\mathrm{K} 6-\mathrm{J} 6$ & $=\mathrm{L} 6 / \mathrm{F} 6$ & $=\mathrm{G} 6$ & $=-1 * \mathrm{M} 6$ & ="*"\&A6 \\
\hline 7 & Eu151 & & & $=\operatorname{AVERAGE}(\mathrm{B} 7: \mathrm{C} 7)$ & & $=\mathrm{E} 7-\mathrm{D} 7$ & Er166 & & & $=\operatorname{AVERAGE}(\mathrm{H7}: \mathrm{I7})$ & & $=\mathrm{K} 7-\mathrm{J} 7$ & $=\mathrm{L} 7 / \mathrm{F} 7$ & $=\mathrm{G} 7$ & $=-1 * \mathrm{M} 7$ & $=" * " \& \mathrm{~A} 7$ \\
\hline 8 & Gd160 & & & $=$ AVERAGE $(B 8: C 8)$ & & $=\mathrm{E} 8$-D8 & Yb172 & & & $=$ AVERAGE(H8:I8) & & $=\mathrm{K} 8-\mathrm{J} 8$ & $=\mathrm{L} 8 / \mathrm{F} 8$ & $=\mathrm{G} 8$ & $=-1 * \mathrm{M} 8$ & $=" * " \& \mathrm{~A} 8$ \\
\hline 9 & Gd160 & & & $=$ AVERAGE $(B 9: C 9)$ & & $=\mathrm{E} 9-\mathrm{D} 9$ & Yb173 & & & $=\operatorname{AVERAGE}(\mathrm{H} 9: 19)$ & & $=$ K9-J9 & $=\mathrm{L} 9 / \mathrm{F} 9$ & $=\mathrm{G} 9$ & $=-1 * \mathrm{M} 9$ & ="*"\&A9 \\
\hline 10 & Ho165 & & & $=$ AVERAGE(B10:C10) & & $=\mathrm{E} 10-\mathrm{D} 10$ & Ta181 & & & $=$ AVERAGE $(\mathrm{H} 10: \mathrm{I} 10)$ & & $=\mathrm{K} 10-\mathrm{J} 10$ & $=\mathrm{L} 10 / \mathrm{F} 10$ & $=\mathrm{G} 10$ & $=-1 * \mathrm{M} 10$ & $=" * " \& \mathrm{~A} 10$ \\
\hline 11 & Er166 & & & $=$ AVERAGE(B11:C11) & & $=$ E11-D11 & W184 & & & $=$ AVERAGE $(\mathrm{H} 11: \mathrm{I} 11)$ & & $=\mathrm{K} 11-\mathrm{J} 11$ & $=\mathrm{L} 11 / \mathrm{F} 11$ & $=\mathrm{G} 11$ & $=-1 * \mathrm{M} 11$ & $=" * " \& \mathrm{~A} 11$ \\
\hline 12 & Ta181 & & & $=$ AVERAGE(B12:C12) & & $=\mathrm{E} 12-\mathrm{D} 12$ & Hf180 & & & $=$ AVERAGE(H12:I12) & & $=\mathrm{K} 12-\mathrm{J} 12$ & $=\mathrm{L} 12 / \mathrm{F} 12$ & $=\mathrm{G} 12$ & $=-1 * \mathrm{M} 12$ & $=" * " \& \mathrm{~A} 12$ \\
\hline 13 & Dy164 & & & $=$ AVERAGE(B13:C13) & & $=\mathrm{E} 13-\mathrm{D} 13$ & Gd160 & & & $=$ AVERAGE(H13:I13) & & $=\mathrm{K} 13-\mathrm{J} 13$ & $=\mathrm{L} 13 / \mathrm{F} 13$ & $=\mathrm{G} 13$ & $=-1 * \mathrm{M} 13$ & $=" * " \& \mathrm{~A} 13$ \\
\hline 14 & Er166 & & & $=$ AVERAGE(B14:C14) & & $=$ E14-D14 & Dy164 & & & $=$ AVERAGE(H14:I14) & & $=\mathrm{K} 14-\mathrm{J} 14$ & $=\mathrm{L} 14 / \mathrm{F} 14$ & $=\mathrm{G} 14$ & $=-1 * \mathrm{M} 14$ & $=" * " \& \mathrm{~A} 14$ \\
\hline 15 & Nd146 & & & $=$ AVERAGE $(\mathrm{B} 15: \mathrm{C} 15)$ & & $=\mathrm{E} 15-\mathrm{D} 15$ & Er166 & & & $=$ AVERAGE $(H 15: I 15)$ & & $=\mathrm{K} 15-\mathrm{J} 15$ & $=\mathrm{L} 15 / \mathrm{F} 15$ & $=\mathrm{G} 15$ & $=-1 * \mathrm{M} 15$ & $=" * " \& \mathrm{~A} 15$ \\
\hline 16 & Dy164 & & & $=$ AVERAGE $(\mathrm{B} 16: \mathrm{C} 16)$ & & $=\mathrm{E} 16-\mathrm{D} 16$ & Hf179 & & & $=$ AVERAGE(H16:I16) & & $=\mathrm{K} 16-\mathrm{J} 16$ & $=\mathrm{L} 16 / \mathrm{F} 16$ & $=\mathrm{G} 16$ & $=-1 * \mathrm{M} 16$ & $=" * " \& \mathrm{~A} 16$ \\
\hline 17 & Gd160 & & & $=$ AVERAGE $(\mathrm{B} 17: \mathrm{C} 17)$ & & $=\mathrm{E} 17-\mathrm{D} 17$ & Lu175 & & & $=$ AVERAGE $($ H17:I17) & & $=\mathrm{K} 17-\mathrm{J} 17$ & $=\mathrm{L} 17 / \mathrm{F} 17$ & $=\mathrm{G} 17$ & $=-1 * \mathrm{M} 17$ & $=" * " \& A 17$ \\
\hline 18 & \multirow{2}{*}{\multicolumn{16}{|c|}{ END FIRST ORDER CORRECTIONS - PUT THESE IN AND REPROCESS DATA FOR SECOND ORDER CORRECTION ELEMENTS LISTED BELOW }} \\
\hline 19 & & & & & & & & & & & & & & & & \\
\hline 20 & \multicolumn{16}{|c|}{ 2ND ORDER CORRECTION ELEMENTS } \\
\hline 21 & Nd146 & & & $=$ AVERAGE(B21:C21) & & $=\mathrm{E} 21-\mathrm{D} 21$ & Gd160 & & & $=$ AVERAGE $(\mathrm{H} 21: \mathrm{I} 21)$ & & $=\mathrm{K} 21-\mathrm{J} 21$ & $=\mathrm{L} 21 / \mathrm{F} 21$ & $=\mathrm{G} 21$ & $=-1 * \mathrm{M} 21$ & $=" * " \& \mathrm{~A} 21$ \\
\hline 22 & $\mathrm{Nd} 146$ & & & $=$ AVERAGE(B22:C22) & & $=\mathrm{E} 22-\mathrm{D} 22$ & Dy164 & & & $=$ AVERAGE $(\mathrm{H} 22: \mathrm{I} 22)$ & & $=\mathrm{K} 22-\mathrm{J} 22$ & $=\mathrm{L} 22 / \mathrm{F} 22$ & $=\mathrm{G} 22$ & $=-1 * \mathrm{M} 22$ & $=" * " \& A 22$ \\
\hline 23 & Sm149 & & & $=$ AVERAGE(B23:C23) & & $=\mathrm{E} 23-\mathrm{D} 23$ & Er166 & & & $=$ AVERAGE $(\mathrm{H} 23: \mathrm{I} 23)$ & & $=\mathrm{K} 23-\mathrm{J} 23$ & $=\mathrm{L} 23 / \mathrm{F} 23$ & $=\mathrm{G} 23$ & $=-1 * \mathrm{M} 23$ & $=" * " \& A 23$ \\
\hline 24 & & & & END SECOND ORDER CC & DRRECTIOI & NS - PUT THE & IN AND REP & OCESS & DATA FC & OR THIRD ORDER COR & RECTION EL & LEMENTS L & ISTED BELO & & & \\
\hline 25 & & & & & & & & & & & & & & & & \\
\hline 26 & \multicolumn{16}{|c|}{ THIRD ORDER CORRECTION ELEMENTS } \\
\hline 27 & Sm149 & & & $=$ =AVERAGE(B27:C27) & & $=\mathrm{E} 27-\mathrm{D} 27$ & Gd160 & & & $=$ =AVERAGE(H27:I27) & & $=\mathrm{K} 27-\mathrm{J} 27$ & $=\mathrm{L} 27 / \mathrm{F} 27$ & $=\mathrm{G} 27$ & $=-1 * \mathrm{M} 27$ & $=" * " \& \mathrm{~A} 27$ \\
\hline 28 & $\operatorname{Sm} 149$ & & & $=$ AVERAGE(B28:C28) & & $=\mathrm{E} 28-\mathrm{D} 28$ & Dy164 & & & $=$ AVERAGE $(\mathrm{H} 28: \mathrm{I} 28)$ & & $=\mathrm{K} 28-\mathrm{J} 28$ & $=\mathrm{L} 28 / \mathrm{F} 28$ & $=\mathrm{G} 28$ & $=-1 * \mathrm{M} 28$ & $=" * " \& \mathrm{~A} 28$ \\
\hline 29 & W184 & & & =AVERAGE(B29:C29) & & $=\mathrm{E} 29-\mathrm{D} 29$ & Hf180 & & & $=$ AVERAGE $(H 29: I 29)$ & & $=\mathrm{K} 29-\mathrm{J} 29$ & $=\mathrm{L} 29 / \mathrm{F} 29$ & $=\mathrm{G} 29$ & $=-1 * \mathrm{M} 29$ & $=" * " \& A 29$ \\
\hline 30 & Dy164 & & & $=$ AVERAGE $(B 30: C 30)$ & & $=\mathrm{E} 30-\mathrm{D} 30$ & Hf180 & & & $=$ AVERAGE $(\mathrm{H} 30: \mathrm{I} 30)$ & & $=\mathrm{K} 30-\mathrm{J} 30$ & $=\mathrm{L} 30 / \mathrm{F} 30$ & $=\mathrm{G} 30$ & $=-1 * \mathrm{M} 30$ & $=" * " \& \mathrm{~A} 30$ \\
\hline 31 & & & & & & & & & & & & & & & & \\
\hline 32 & & & & & & & & & & & & & & & & \\
\hline 33 & \multicolumn{16}{|c|}{ OTHER OXIDE CORRECTIONS } \\
\hline 34 & $\mathrm{Ca} 43$ & & & $=$ AVERAGE(B34:C34) & & $=$ E34-D34 & Fe57 & & & $=$ =AVERAGE(H34:I34) & & $=\mathrm{K} 34-\mathrm{J} 34$ & $=\mathrm{L} 34 / \mathrm{F} 34$ & $=\mathrm{G} 34$ & $=-1 * \mathrm{M} 34$ & $=" * " \& \mathrm{~A} 34$ \\
\hline 35 & $\mathrm{Ca} 43$ & & & =AVERAGE(B35:C35) & & $=\mathrm{E} 35-\mathrm{D} 35$ & Co59 & & & $=$ AVERAGE $($ H35:I35) & & $=\mathrm{K} 35-\mathrm{J} 35$ & $=\mathrm{L} 35 / \mathrm{F} 35$ & $=\mathrm{G} 35$ & $=-1 * \mathrm{M} 35$ & $=" * " \& A 35$ \\
\hline 36 & $\mathrm{Ca} 43$ & & & $=$ AVERAGE $(B 36: C 36)$ & & $=\mathrm{E} 36-\mathrm{D} 36$ & Ni60 & & & $=$ AVERAGE $(H 36: I 36)$ & & $=\mathrm{K} 36-\mathrm{J} 36$ & $=\mathrm{L} 36 / \mathrm{F} 36$ & $=\mathrm{G} 36$ & $=-1 * \mathrm{M} 36$ & $=" * " \& \mathrm{~A} 36$ \\
\hline 37 & & & & & & & & & & & & & & & & \\
\hline 38 & $\mathrm{Zr} 90$ & & & $=$ AVERAGE(B38:C38) & & $=\mathrm{E} 38-\mathrm{D} 38$ & Ag107 & & & $=$ AVERAGE $($ H38:I38) & & $=\mathrm{K} 38-\mathrm{J} 38$ & $=\mathrm{L} 38 / \mathrm{F} 38$ & $=\mathrm{G} 38$ & $=-1 * \mathrm{M} 38$ & $=" * " \& \mathrm{~A} 38$ \\
\hline 39 & $\mathrm{Nb} 93$ & & & =AVERAGE(B39:C39) & & $=$ E39-D39 & Ag109 & & & $=$ AVERAGE(H39:I39) & & $=\mathrm{K} 39-\mathrm{J} 39$ & $=\mathrm{L} 39 / \mathrm{F} 39$ & $=\mathrm{G} 39$ & $=-1 * \mathrm{M} 39$ & ="*"\&A39 \\
\hline $\begin{array}{l}40 \\
41\end{array}$ & Mo 95 & & & $=$ AVERAGE $(B 41: \mathrm{C} 41)$ & & $=$ E41-D41 & $\mathrm{Cd} 111$ & & & $=$ AVER AGE $(\mathrm{H} 41: \mathrm{I} 41)$ & & $=\mathrm{K} 41-\mathrm{J} 41$ & $=\mathrm{L} 41 / \mathrm{F} 41$ & $=\mathrm{G} 41$ & $=-1 * \mathrm{M} 41$ & $=" * " \& A 41$ \\
\hline 42 & Mo 98 & & & $=$ AVERAGE $(\mathrm{B} 42: \mathrm{C} 42)$ & & $=\mathrm{E} 42-\mathrm{D} 42$ & $\mathrm{Cd} 114$ & & & $=$ AVERAGE(H42:I42) & & $=\mathrm{K} 42-\mathrm{J} 42$ & $=\mathrm{L} 42 / \mathrm{F} 42$ & $=\mathrm{G} 42$ & $=-1 * \mathrm{M} 42$ & $=" * " \& A 42$ \\
\hline
\end{tabular}

